Revue québécoise de droit international

Quebec Journal of International Law

Revista quebequense de derecho internacional

\title{
Entre théories et pratiques : la nature, sujet de droit dans la constitution équatorienne, considérations critiques sur une vieille antienne
}

\section{Rabah Belaidi}

Numéro hors-série, septembre 2018

Terre à terre : environnement et approches critiques du droit

URI : https://id.erudit.org/iderudit/1067015ar

DOI : https://doi.org/10.7202/1067015ar

Aller au sommaire du numéro

\section{Éditeur(s)}

Société québécoise de droit international

ISSN

0828-9999 (imprimé)

2561-6994 (numérique)

Découvrir la revue

Citer cet article

Belaidi, R. (2018). Entre théories et pratiques : la nature, sujet de droit dans la constitution équatorienne, considérations critiques sur une vieille antienne. Revue québécoise de droit international / Quebec Journal of International Law / Revista quebequense de derecho internacional, 93-124.

https://doi.org/10.7202/1067015ar

\section{Résumé de l'article}

La question de l'octroi de la personnalité juridique à la nature est une idée qui traverse les fondements du droit de l'environnement depuis plus de quarante ans. Le thème connaît un regain d'intérêt au sein de la doctrine juridique de divers systèmes de droits et présente une acuité particulière au vu des expériences en cours de réalisation en Amérique du Sud. Une traduction juridique a été donnée par la Constitution équatorienne de 2008 qui a érigé la nature comme un sujet de droit à part entière, annonçant ainsi une grande " première ". La présente contribution analyse les évolutions juridiques ainsi que les conceptions théoriques qui sous-tendent ces changements constitutionnels. Sans prétendre à une évaluation du droit et des décisions de justice postérieures à l'entrée en vigueur de la constitution, cet article entend faire l'apport de critiques sur l'octroi de la qualité de sujet à la nature. Insérée dans un contexte théorique syncrétique mêlant références à des notions mythiques amérindiennes, systèmes contemporains de résolution des conflits et conceptions post-modernes de l'interprétation constitutionnelle, l'attribution de la personnalité juridique à la nature est indissociable du nouveau constitutionnalisme latino-américain. Il apparaît que, loin de pouvoir illustrer une révolution du ou dans le droit, la Constitution équatorienne et l'application qui en est faite par les tribunaux ne démontrent pas disposer d'un degré de protection supérieur aux systèmes dits " classiques ". Le pouvoir politique à l'origine de la création de la Constitution montre également les limites des conceptions supposément nouvelles face à des configurations de logique d'économie globalisée et d'intérêts à préserver.
Tous droits réservés (C Société québécoise de droit international, 2018

Ce document est protégé par la loi sur le droit d'auteur. L'utilisation des services d'Érudit (y compris la reproduction) est assujettie à sa politique d'utilisation que vous pouvez consulter en ligne.

https://apropos.erudit.org/fr/usagers/politique-dutilisation/ 


\title{
ENTRE THÉORIES ET PRATIQUES : LA NATURE, SUJET DE DROIT DANS LA CONSTITUTION ÉQUATORIENNE, CONSIDÉRATIONS CRITIQUES SUR UNE VIEILLE ANTIENNE
}

\author{
Rabah Belaidi ${ }^{*}$
}

La question de l'octroi de la personnalité juridique à la nature est une idée qui traverse les fondements du droit de l'environnement depuis plus de quarante ans. Le thème connaît un regain d'intérêt au sein de la doctrine juridique de divers systèmes de droits et présente une acuité particulière au vu des expériences en cours de réalisation en Amérique du Sud. Une traduction juridique a été donnée par la Constitution équatorienne de 2008 qui a érigé la nature comme un sujet de droit à part entière, annonçant ainsi une grande «première». La présente contribution analyse les évolutions juridiques ainsi que les conceptions théoriques qui sous-tendent ces changements constitutionnels. Sans prétendre à une évaluation du droit et des décisions de justice postérieures à l'entrée en vigueur de la constitution, cet article entend faire l'apport de critiques sur l'octroi de la qualité de sujet à la nature. Insérée dans un contexte théorique syncrétique mêlant références à des notions mythiques amérindiennes, systèmes contemporains de résolution des conflits et conceptions post-modernes de l'interprétation constitutionnelle, l'attribution de la personnalité juridique à la nature est indissociable du nouveau constitutionnalisme latino-américain. Il apparaît que, loin de pouvoir illustrer une révolution du ou dans le droit, la Constitution équatorienne et l'application qui en est faite par les tribunaux ne démontrent pas disposer d'un degré de protection supérieur aux systèmes dits «classiques». Le pouvoir politique à l'origine de la création de la Constitution montre également les limites des conceptions supposément nouvelles face à des configurations de logique d'économie globalisée et d'intérêts à préserver.

The question of whether to grant nature a legal personality is an idea that has been coursing through the foundations of environmental law for more than forty years. There has been renewed interest in this theme within the legal doctrine of various rights systems, and is becoming particularly acute in light of the current experiences in South America. A legal translation was given by the 2008 Constitution of Ecuador, which erected nature as a full subject of law, constituting a historical first. This contribution analyses the legal evolutions as well as the theoretical conceptions underlying these constitutional changes. Without purporting to be an evaluation of the law and court decisions following the entry into force of the Constitution, this article seeks to contribute critiques on granting nature the status of subject of law. Inserted within a syncretic theoretical context, blending references to Indigenous mythical notions, contemporary dispute resolution systems, and post-modern conceptions of constitutional interpretation, the granting of legal personality to nature is inseparable from the new Latin American constitutionalism. It appears that, far from being able to illustrate a revolution of, or within, law, the Constitution of Ecuador and its application by courts do not demonstrate a higher degree of protection in comparison to so-called "classical" systems. The political power from which stems the creation of the Constitution also shows the limits of supposedly new conceptions towards configurations related to a globalized economy logic and interests to preserve.

La cuestión de otorgar personalidad jurídica a la naturaleza es una idea que ha sustentado las bases del derecho ambiental durante más de cuarenta años. El tema está ganando un renovado interés en la doctrina legal de varios sistemas de derechos y es particularmente relevante a la luz de las experiencias en curso en América del Sur. Una traducción legal fue dada por la Constitución Ecuatoriana de 2008 que estableció la naturaleza como un sujeto de derechos pleno, anunciando así un gran "estreno". Este trabajo analiza los desarrollos legales, así como las concepciones teóricas que subyacen a estos cambios constitucionales. Sin pretender una evaluación de la ley y las decisiones judiciales después de la entrada en vigor de la

* Professeur titulaire, Faculté de droit de l’Université Fédérale de Goiás (Brésil). Docteur en droit de l’Université Panthéon Assas (Paris). 
constitución, este artículo tiene la intención de traer críticas sobre la concesión de la calidad de sujeto a la naturaleza. Insertada en un contexto teórico sincrético mezclando referencias a nociones míticas nativo americanas, sistemas de resolución de conflictos contemporáneos y concepciones posmodernas de interpretación constitucional, la atribución de personalidad jurídica a la naturaleza es inseparable del nuevo constitucionalismo latinoamericano. Parece que, lejos de poder ilustrar una revolución en el derecho o en la ley, la Constitución ecuatoriana y la aplicación de los tribunales no demuestran que tengan un grado de protección más alto que los sistemas llamados "clásicos". El poder político detrás de la creación de la Constitución también muestra los límites de las ideas supuestamente nuevas frente a configuraciones de lógica de economía globalizada y de intereses a preservar. 
Lorsqu'on s'interroge sur les aspects théoriques d'une branche du droit ou même d'une discipline, les différents niveaux de discours mobilisables en faveur ou contre un certain type d'arguments doivent, nous semble-t-il, être précisés avec une particulière acuité. Cette présente contribution n'échappe pas à la règle pour une série de motifs. En premier lieu, le contenu de l'article traite essentiellement d'un droit national étranger (le droit équatorien) et pourrait légitimement provoquer l'étonnement et la surprise du juriste internationaliste, davantage préoccupé par l'agencement et la possible critique de dispositifs juridiques internationaux (traités, coutumes, résolutions) ou de décisions, contraignantes ou non, prononcées par des commissions, cours ou tribunaux. Il n'empêche que si l'on s'enquiert sur les fondements théoriques $\mathrm{du}$ droit de l'environnement, tant au niveau national qu'international, l'une des questions actuellement (re)débattues tient à l'attribution de la personnalité juridique à la nature. Considérer cette dernière comme un sujet de droit n'est pas une question nouvelle. Le débat fut lancé de manière mémorable aux États-Unis en 1971 par Christopher Stone, qui dans un article au titre provocateur suggérait la nécessité de considérer les arbres comme des sujets à part entière ${ }^{1}$. Après s'être atténuée, la question semble à nouveau préoccuper tant la doctrine juridique de divers systèmes de droits ${ }^{2}$ que les philosophes du droit ${ }^{3}$. Du monde parfois clos des juristes académiques, dont la production de propositions et d'élaborations conceptuelles théoriques n'a pas manqué, nous sommes passés ces dernières années à des expériences qui intéressent les observateurs de tous bords : opérateurs juridiques et praticiens du droit, juristes dogmatiques, théoriciens du droit, défenseurs militants et activistes de la cause environnementale et/ou animale ${ }^{4}$. Le moment est effectivement centré sur les pratiques, puisque progressivement divers ordonnancements juridiques (nationaux et internationaux) s'évertuent à accroître notablement les degrés de protection de la nature. Il convient d'ailleurs de remarquer

1 Christopher D Stone, «Should Trees Have Standing? Towards Legal Rights for Natural Objects » (1972) 45:2 S Cal L Rev 450. L'auteur a actualisé sa thèse. Voir Christopher D Stone, Should Trees Have Standing? Law, Morality, and the Environment, Oxford, Oxford University Press, 2010.

2 Jean-Pierre Marguénaud, «La personnalité juridique des animaux » (1998) 20 D 205; Marie-Angèle Hermitte, «La nature, sujet de droit?» (2011) 66:1 Annales. Histoire, Sciences Sociales 173; Serge Gutwirth, «Trente ans de théorie du droit de l'environnement: concepts et opinions » (2001) 26 Environnement et Société 5; Alexandre Zabalza, La terre, le droit : Du droit civil à la philosophie du droit, Pompignac, Bière, 2007; Lynda Warren, dir, Wild law : Is There any Evidence of Earth Jurisprudence in Existing Law and Practice? An International Research Project, Londres, Environmental Law Association et The Gaia Foundation, 2009.

3 Lukas Sosoe, «D'un prétendu droit de la nature: Trois hypostases » (1992) 22 Cahiers de Philosophie politique et juridique de l'Université de Caen 183 [Sosoe]; François Ost, La nature hors la loi, l'écologie à l'épreuve du droit, Paris, Découverte, 1995 [Ost].

4 La question dépasse le monde du droit et des juristes. Depuis les deux dernières décennies des philosophes et autres spécialistes en sciences sociales ne manquent pas de s'intéresser au débat sur le type d'écologie nécessaire ou souhaitable aux sociétés modernes. Entre écologie profonde, éthique de la nature, anthropocentrisme ou biocentrisme, les courants et appelations foisonnent. Voir Marcel Gauchet, «Sous l'amour de la nature, la haine des hommes » (1990) 3:60 Le Débat 247; Luc Ferry, Le nouvel ordre écologique : L'arbre, l'animal et l'homme, Paris, Bernard Grasset, 1992. Plus récemment voir Bruno Latour, Face à Gaïa: Huit conférences sur le Nouveau Régime Climatique, Paris, Les Empêcheurs de penser en rond et Découverte, 2015 [Latour]; John Baird Callicot, Thinking Like a Planet: The Land Ethic and the Earth Ethic, New York, Oxford University Press, 2014; John Baird Callicot, Ethique de la terre, Paris, Wildproject, 2010. 
à cet égard qu'il n'y a pas a priori de différence sui generis entre droit national et international. Par conséquent un thème de droit interne peut a priori relever un certain intérêt pour le droit international. Tel est le cas de la personnification juridique de la nature, dont les intérêts pour les débats sur la consolidation, l'avenir ou l'effectivité du droit international de l'environnement semblent pertinents.

En droit national, ces expériences correspondent peu ou prou à la prétendue alternative biocentrisme/anthropocentrisme. Loin de vouloir tomber dans les travers d'une dualité fermée ${ }^{5}$, puisque d'autres voies sont toujours possibles et souhaitables, tant d'un point de vue académique que politique, notre article ne souhaite pas prendre partie à ce débat entre anthropocentrisme et biocentrisme. Il prétend surtout dévoiler les ressorts de propositions et solutions qui nous semblent, non seulement en contradiction avec leur caractère de nouveauté, mais aussi fausses sur le plan du fonctionnement du droit et des résultats qui peuvent en être attendus. Les expériences d'ordonnancements juridiques qualifiés d'anthropocentriques sont ultra-majoritaires au sein de différents systèmes de droit et il revient au droit comparé de nous révéler les divergences ou convergences d'approches, notamment en termes d'efficacité ou d'effectivité. Il n'en va pas de même au regard des pratiques juridiques biocentriques. Il existe très peu de cas de personnification juridique de la nature. L'une d'entre elles a eu lieu en Nouvelle-Zélande où l'octroi de la personnalité juridique fut limité à une rivière et à un ex-parc national, suivi du cas du fleuve indien Gange et de son affluent Yamuna $^{6}$. L'autre a pris place en Équateur et de manière plus générale, plus solennelle, puisque la nouvelle Constitution adoptée en 2008 a reconnu de manière

5 Pierre Bourdieu s'insurge contre ces manifestations de la pensée faites de dualités antangoniques (interne/externe, pure/impur, normatif/positif, axiologique/sociologique, compréhensif/explicatif, Kelsen et Marx) qu'il qualifie de calamités des sciences sociales. Pierre Bourdieu, « Les juristes, gardiens de l'hypocrisie collective » dans François Chazel et Jacques Commaille, dir, Normes juridiques et régulation sociale, Paris, LGDJ, 1991, 95.

6 La rivière Whanganui et parc national Te Urewera, sont dénommés aujourd'hui simplement Te Urewera. Voir Catherine J Iorns Magallanes, « Nature as an Ancestor: Two Examples of Legal Personality for Nature in New Zealand » (2015) hors-série 22 VertigO, La revue électronique en sciences de l'environnement, en ligne: <vertigo.revues.org/16199>. La Cour suprême de l'État de l'Uttarakhand en Inde a reconnu en mars 2017 le Gange et l'un de ses affluents, la rivière Yamuna comme des entités vivantes détentrices de la personnalité morale. L'aspect le plus important selon nous est l'obligation faite à l'État de l'Uttarakhand de créer dans le délai de trois mois un « management board» constitué de représentants spécifiques (différent des tuteurs pouvant exercer le droit de protection) ayant pour charge de proposer et d'appliquer des solutions de protection du fleuve et de son affluent. Mohd. Salim (Petitioner) v State of Uttarakhand \& others, 2017 High Court of Uttarakhand at Nainital Writ Petition (Pil) n 126 2014. En Nouvelle-Calédonie, les îles Loyauté qui forment l'une des trois provinces, a adopté en 2016 un code de l'environnement contenant des principes généraux. L'article 110-3 dispose ainsi que : « Le principe unitaire de vie qui signifie que l'homme appartient à l'environnement naturel qui l'entoure et conçoit son identité dans les éléments de cet environnement naturel constitue le principe fondateur de la société kanak. Afin de tenir compte de cette conception de la vie et de l'organisation sociale kanak, certains éléments de la Nature pourront se voir reconnaitre une personnalité juridique dotée de droits qui leur sont propres, sous réserve des dispositions législatives et réglementaires en vigueur ». Province des Îles Loyauté (Nouvelle Calédonie), Délibération $n^{\circ} 2016$ 13/API du 6 avril 2016 portant adoption du Code de l'environnement de la province des îles Loyauté, JONC, 23 juin 2016, 5936 aux pp 5941-42. Ce code n'est encore que très parcellaire puisque bon nombre de ses dispositions (livres et titres) ont été réservées et n'ont donc pas encore été rédigées, discutées et votées. 
emphatique la nature comme un sujet de $\operatorname{droit}(\mathrm{s})$. Le contexte politique et juridique latino-américain de ces dernières années est propice à d'intenses discussions théoriques sur de nombreux thèmes de société : consolidation de la démocratie au moyen, notamment, de nouvelles formes participatives, d'une protection accrue des droits de l'homme et des peuples indigènes, et d'une volonté d'instaurer des États plurinationaux. Les thèmes ne manquent pas et pour ce qui a trait à leurs traductions juridiques, le débat théorique a été mené à partir des thèses du néo ou nouveau constitutionnalisme. Au sein de cette effervescence constitutionnelle, deux pays, l'Équateur et la Bolivie ${ }^{7}$, se distinguent quant à l'importante place accordée aux thèmes de l'indianité ou d'un certain « indigénisme ${ }^{8}$ » juridique. Cette attention portée à ces derniers n'est pas seulement de les concevoir en tant que catégories de populations à protéger au moyen du droit, mais aussi, et surtout, par une affirmation politique et constitutionnelle de ces États de considérer que le droit et l'organisation de la société se doivent d'être orientés et déterminés à partir de préceptes amérindiens/indigènes de nature mythique. Ainsi apparaissent dans la Constitution équatorienne à de multiples reprises, de façon centrale et cardinale les termes pachamama ou sumak kawsay ${ }^{9}$ qui sont érigés comme des concepts pourvus d'une certaine normativité, puisque logés dans la Constitution et ayant pour ambition de servir de référence à l'interprétation de tous les autres énoncés constitutionnels. Puisés dans une vision cosmologique des peuples andins, ces concepts, flous ${ }^{10}$ pour le juriste formé à la modernité de l'enseignement des facultés de droit, sont censés apporter de nouveaux fondements pour la compréhension de la vie, de la nature et des

7 La Constitution bolivienne de 2009 s'inscrit globalement dans le même contexte socio-politique et juridique de l'Équateur, mais la nature n'a pas été formellement reconnue comme un sujet de droit à part entière. Sans nul doute, la situation bolivienne est propice aux recherches juridiques car riche de connaissances qui ont déjà suscité, à juste titre, bon nombre de commentaires et analyses. Il nous a cependant paru nécessaire dans le cadre de notre débat de devoir nous limiter à la Constitution équatorienne eu égard à la force symbolique, juridique et politique que constituent l'attribution de la personnalité juridique à la nature. D'autres recherches doivent suivre, y compris sur un terrain comparatif, entre ces deux constitutions, eu égard aux profondes ressemblances et à l'existence de dissemblances. Eduardo Gudynas établit un grand nombre de différences d'approches entre les constitutions de ces deux États. Eduardo Gudynas, « Développement, droits de la Nature et Bien Vivre : l'expérience équatorienne » (2011) 4:68 Mouvements 15 à la p 23.

8 Le terme " indigénisme » présente diverses acceptions, parfois même contradictoires dans leur usage. Il peut désigner un courant littéraire et artistique latino-américain à la recherche de ses racines indiennes. D'un point de vue politique, l'indigénisme peut être caractérisé à partir de trois éléments : « la dénonciation de l'oppression de l'Indien; la recherche de politiques permettant de dépasser la problématique indigène en intégrant l'Indien à l'ensemble de la société; et, découlant de ce qui précède, la manifestation du caractère métis, indo-américain, du continent. Il s'agit d'un programme à la fois de dénonciation et d'auto-affirmation : sauver l'indigène pour affirmer une identité complexe, propre, autonome et différente de la culture occidentale. C'est pour cette raison que l'indigénisme a été et est toujours avant tout une idéologie de non-Indiens, bien qu'il y ait eu de nombreux indigènes indigénistes ", José Bengoa, «Violence et émergence de l'indianité en Amérique » (2003) 26 Ateliers 109 au para 60.

9 Pachamama signifie en espagnol madre tierra, la terre mère. Sumak Kawsay est traduit comme buen vivir, le bon vivre ou bien-être.

10 On se heurte à un problème de signification et l'on vient à s'en demander finalement s'ils n'étaient pas des synonymes ou des variantes indigènes des termes développement, qualité de vie, bien-être, nature, ou environnement. 
droits ${ }^{11}$. La volonté clairement assumée est de provoquer une rupture avec l'héritage de la colonisation espagnole ainsi que des épistémologies occidentales, souvent qualifiées d'eurocentriques. Il n'empêche qu'à la lecture claire et simple de la Constitution équatorienne, on est frappé par le caractère syncrétique des dispositions qu'elle contient. Qu'on le veuille ou non, les énoncés constitutionnels reposent avant tout sur des techniques, mécanismes issus de la modernité. Il en va à l'évidence de la qualité de sujet de droit qui est l'incarnation d'une vision moderne, occidentale et dogmatique du droit ${ }^{12}$. Et il nous importe finalement de savoir si la personnification juridique de la nature apporte, par rapport aux systèmes dits anthropocentriques, des modifications juridiques substantielles dans la protection offerte à l'environnement et si oui, de quelle manière?

Nous tenons pour hypothèse que la qualité de la protection offerte à la nature, si elle se définit comme étant l'efficacité, l'effectivité ou l'efficience du droit de l'environnement, n'est pas tributaire de la personnification juridique de celle-ci. Autrement dit, il n'est pas prouvé, bien que le sens commun d'une grande partie des juristes tente de le consacrer, que la consécration de la qualité de sujet à la nature lui fait bénéficier d'un régime ipso facto plus avantageux quant à sa protection, conservation et préservation. Si nous nous autorisons telle hypothèse, c'est à partir d'un cadre théorique et épistémologique de conception réaliste qui conçoit le droit comme étant ce que font les opérateurs juridiques authentiques. Et que l'application du droit n'est jamais détachable du travail d'interprétation des énoncés juridiques.

Notre article n'a pas l'ambition de montrer ou esquisser ce qui serait une troisième voie, c'est-à-dire adopter un discours prescriptif. Il prétend davantage analyser les propos et arguments de théories du droit intimement liées à un projet biocentrique, en insistant tout particulièrement sur une description réelle de ses pratiques telles qu'elles sont appliquées par ses interprètes authentiques (juges, fonctionnaires, législateurs). Notre recherche ne prétend pas assumer un aspect partisan au sein du débat bio ou anthropocentrisme ou se positionner pour ou contre l'attribution de la personnalité juridique à la nature, mais plutôt d'éclaircir que ce débat est tributaire d'une théorie(sation) du droit. On ne peut faire l'économie d'un replacement de cette discussion à partir d'une analyse métathéorique qui permet à son tour de cerner avec plus d'acuité les conditions dans lesquelles la personnalité juridique prétend s'insérer comme une solution. Pour ce faire, il nous paraît nécessaire dans une première partie de s'intéresser au nouveau cadre théorique revendiqué par le mouvement du néo ou nouveau constitutionnalisme et pour lequel la Constitution équatorienne représente un objet empirique pertinent. Nous aurions pu, à la manière de Pierre Bourdieu, vouloir partir des pratiques pour ensuite pouvoir

11 Vitor Sousa Freitas, « Os Novos Direitos da Natureza : Horizontes a Conquistar » dans Antonio Carlos Wolkmer et José Rubens Morato Leite, dir, Os «novos" direitos no Brasil »: Natureza e Perspectivas Uma visão básica das novas conflituosidades jurídicas, $3^{\mathrm{e}}$ ed, São Paulo, Saraiva, 2016, 287 [Freitas].

12 Un premier paradoxe apparait puisque la qualité de sujet de droit qui est le fruit d'une éminente conception et construction historique d'un droit bourgeois, est l'enjeu de revendications et d'appropriations de la part d'instruments juridiques se voulant révolutionnaires ou favorables à un retour pré-moderne. 
théoriser une réalité ${ }^{13}$, mais la réalité qui s'offre à nous est justement inverse. La théorie juridique constitutionnelle supposée nouvelle prétend innover les pratiques et ajuster les comportements de manière radicalement différente quant à l'idée de droit, tel que classiquement défini. La deuxième partie de notre article procédera à l'analyse des expériences observées depuis l'entrée en vigueur de la constitution, afin de pouvoir porter un premier regard, nécessairement critique sur la façon dont la théorie aurait permis de favoriser l'éclosion de nouvelles pratiques judiciaires et voir en fin de compte si les pratiques ont pu déceler ou confirmer de supposées nouvelles théories $^{14}$.

\section{Constitutionnalisation et personnification de la nature : contexte politique et théorie du droit}

Nous entendons nous limiter à une approche contextuelle ${ }^{15}$ politique afin de souligner l'aspect sociopolitique et historique du nouveau constitutionnalisme (A). Les aspects relevant de la mécanique de la théorie du droit, de type interne (hors contexte), seront présentés de manière globale, dans leur diversité, tels qu'ils apparaissent dans leur mobilisation argumentative, illustrant ainsi le magma de thèses, théories, interprétations et doctrines qui caractérisent le nouveau constitutionnalisme ${ }^{16}$ (B).

\section{A. Une nouvelle constitution : entre texte et contexte}

Le contexte de l'adoption de la Constitution est intimement lié à la période de re-démocratisation de l'Amérique latine. Depuis les années 80, la plupart des États engagent des processus de sortie de régime de type dictatorial. L'adoption de nouvelles constitutions s'inscrit ainsi dans cette logique de re-démocratisation sur fond de critique du système représentatif et «d'une vive remise en question du modèle représentatif, et plus généralement de l'insuffisante démocratisation de l'accès au pouvoir politique pour une grande partie des populations indiennes ${ }^{17} »$.

13 Pierre Bourdieu, Esquisse d'une théorie de la pratique, Genève, Droz, 1972.

14 Il convient de signaler que les sources documentaires du contentieux produit par les tribunaux et cours équatoriennes, ainsi que l'activité de jurisprudence de la Cour suprême sont tributaires de leur mise à disposition. Notre étude ne repose que sur les décisions qui ont été rendues publiques par ces autorités et qui se présentent de manière parcellaire au chercheur.

15 Pour une compréhension plus précise de l'étude du droit en contexte, il convient de consulter Mark Van Hoecke, « Le droit en contexte » (2013) 1:70 Rev interdiscipl ét jur 274.

16 Nous assimilerons pour des raisons de convenance les expressions néo et nouveau constitutionalisme, ainsi que le constitutionnalisme latino-américain et autres expressions voisines, qui n'en sont que des versions ne présentant que de subtiles différences. Il y a toutefois, pour les adeptes et défenseurs de ces thèses, certaines différences de degré ou d'approche, qui ne nous paraissent pas constater l'existence de profondes différences théoriques.

17 Julie Massal, «L'incertaine construction de l'État plurinational et de la démocratie participative en Équateur : aléas et résistances » (2013) 20:1 Revue internationale de politique comparée 117 à la p 117 . 
Néanmoins, ces phases de re-démocratisation ne sont pas toutes passées par l'adoption de textes constitutionnels liés à la doctrine du néo-constitutionnalisme. Seules les constitutions du Venezuela (1999), de l'Équateur (2008) et de la Bolivie (2009) seraient l'apanage des théories du nouveau constitutionnalisme. Cette théorie relève, en effet, de deux éléments, certes souvent complémentaires, mais non nécessairement cumulatifs ou consécutifs. L'adoption de nouvelles constitutions ne signifie pas obligatoirement une adhésion aux thèses néo-constitutionnalistes et vice et versa : cette nouvelle vision constitutionnelle qui laisse une large place au juge (notamment suprême) s'accommode de textes moins récents (notamment dans le cas du Brésil) ${ }^{18}$.

Dans ce contexte les constitutions de l'Équateur et de la Bolivie vont se distinguer quant à leurs approches des thèmes de protection de la nature et de revalorisation des communautés amérindiennes ${ }^{19}$, non plus considérées comme des minorités, mais comme sujets juridiques à part entière, dans un État qualifié de plurinational. Le président de l'Assemblée constituante de l'Équateur, Alberto Acosta, ainsi que l'environnementaliste uruguayen, Eduardo Gudynas, sont généralement connus pour avoir été les idéalisateurs, inspirateurs et même chevilles ouvrières du texte constitutionnel. Le buen vivir de concert avec l'attribution de droits subjectifs à la nature sont censés constituer une approche différente du développement classique $^{20}$. Toute l'idée est là, en cours de construction, comme les deux auteurs le rappelèrent dans de nombreux articles de 2008 à $2013^{21}$.

L'étude de la structure du texte constitutionnel équatorien révèle le contexte de re-démocratisation, les espoirs placés dans la règle de droit, à son plus haut degré. En effet, la Constitution équatorienne de 2008 est un texte «lourd » de 444 articles où le mot nature, pris au sens du mot environnement, apparaît une trentaine fois et tout particulièrement au début du préambule: «le peuple souverain de l'Équateur: [...] célébrant la Nature, la Pachamama, de laquelle nous sommes parts et qui est vitale pour notre existence ${ }^{22} \gg$. Au sein du titre 2 consacré aux droits, le chapitre premier traite des principes d'application des droits et établit très clairement que la

18 Voir ci-dessous, le chapitre I(B) du présent article « Un nouveau constitutionnalisme? Entre nouveaux textes et nouveaux pouvoirs du juge $»$.

$1955 \%$ de la population bolivienne et $40 \%$ de la population équatorienne sont amérindiens-indigènes.

20 Eduardo Gudynas et Alberto Acosta, « La renovación de la crítica al desarrollo y el buen vivir como alternativa: Utopía y Praxis Latinoamericana»(2011) 16:53 Revista Internacional de Filosofía Iberoamericana y Teoría Social 71.

21 Alberto Acosta a rompu politiquement avec la ligne politique du président Rafael Correa à cause de désaccords sur la protection de la nature et sur la permanence d'un projet néo-extractiviste comme matrice au développement. Après avoir été le président de l'Assemblée constituante, puis ministre des mines et de l'énergie, il s'est présenté contre Rafael Correa à la présidence de l'Équateur en 2013. Alberto Acosta, «Why Ecuador's President Has Failed the Country Over Yasuní-ITT » (4 septembre 2013), The Guardian, en ligne : <www.theguardian.com/global-development/2013/sep/04/ecuadorpresident-failed-country-yasuni-itt $>$. Voir aussi Eduardo Gudynas et Alberto Acosta, «El buen vivir o la disolución de la idea del progreso » dans Mariano Rojas, dir, La Medición del Progreso y del Bienestar. Propuestas desde América Latina, AC, México DF, Foro Consultivo Científico y Tecnológico, 2011.

22 Constitución de la república del Ecuador, préambule, Assamblea Constituyente, 2008 [Constitución del Ecuador]. 
« nature sera sujet des droits que la Constitution reconnait ${ }^{23}$ ». Enfin, le chapitre 8 du même titre est consacré aux droits de la nature. Il prévoit par une série de 4 articles (71 à 74) l'essentiel, mais non l'intégralité, des dispositions normatives subjectives (au sens de droits subjectifs, c'est à dire de prérogatives reconnues à un sujet de droit). Pour une meilleure compréhension des termes et enjeux du débat, il nous paraît nécessaire de les citer et de les présenter traduits ${ }^{24}$ :

Art. 71.- La nature ou Pachamama, où se reproduit et se réalise la vie, a droit au respect intégral de son existence et du maintien et de la régénération de ses cycles vitaux, de structure, fonctions et processus évolutifs.

Toute personne, communauté, peuple ou nationalité pourra exiger de l'autorité publique l'accomplissement des droits de la nature. Pour appliquer et interpréter ces droits, il faut observer les principes établis dans la Constitution qui s'y rapportent ${ }^{25}$.

L'État incitera les personnes physiques, juridiques et les collectifs à protéger la nature et promouvoir le respect de tous les éléments qui forment un écosystème.

Art. 72.- La nature a droit à la restauration. Cette restauration sera indépendante de l'obligation qu'ont les États et les personnes physiques ou juridiques d'indemniser les individus et collectifs qui dépendent des systèmes naturels affectés.

Dans les cas d'impact grave et permanent sur l'environnement, y compris ceux causés par l'exploitation des ressources naturelles non renouvelables, l'État établira les mécanismes les plus efficaces afin d'arriver à une restauration et adoptera les mesures adéquates afin d'éliminer ou d'atténuer les conséquences nocives sur l'environnement.

Art. 73.- L'État appliquera des mesures de précaution et de restriction pour les activités pouvant conduire à l'extinction d'espèces, la destruction d'écosystèmes ou l'altération permanente des cycles naturels.

Est prohibée l'introduction d'organismes et de matériel organique et inorganique pouvant altérer de manière définitive le patrimoine génétique national.

Art. 74.- Les personnes, communautés, peuples et nationalités auront droit

23 Ibid, art 10.

24 La traduction de l'ensemble des articles de la Constitution, de textes de lois, de sentences ou d'articles de revues doctrinales en langue espagnole est le fruit de notre traduction.

25 «se observaran los principios establecidos en la Constitución, en lo que proceda», que nous traduisons par «il faut observer les principes établis dans la Constitution qui s'y rapportent ». La signification des mots «en lo que proceda» montre, au-delà de tout problème de traduction, le caractère profondément indéterminé et la pluralité des sens possibles, que ce soit en espagnol (langue originale de la constitution) ou en français (langue de notre traduction). C'est dire que si le droit est intimement lié à l'idée de litige, il faut également dire combien il est un langage et donc nécessairement soumis à l'interprétation des agents locuteurs. Ce simple exemple tiré d'à peine quatre mots espagnols de la Constitution montre le défi auquel sont confrontés les agents juridiques (citoyens, entreprises, administrations publiques et tribunaux), quant au périmètre des règles mobilisables. 
au bénéfice de l'environnement et des richesses naturelles que leur permettent le buen vivir. Les services environnementaux ne seront pas susceptibles d'appropriation; leur production, prestation, utilisation et profit seront régulés par l'État ${ }^{26}$. [Notre traduction.]

À côté de l'attribution de droits subjectifs, conçus comme de véritables prérogatives, la nature est également une référence pour d'autres dispositifs issus de cette même constitution, mais à partir d'agencements différents et souvent, quoique non exclusivement, indirects. On peut se risquer à une esquisse de typologie. Ces dispositifs différents peuvent en effet apparaître de diverses manières. En premier lieu, ils se présentent comme des droits (subjectifs ?) attribués aux citoyens, comme le dispose l'article 66.27 du chapitre 6 consacré aux droits de liberté : « Sont reconnus et garantis aux personnes [...] le droit à vivre dans un environnement sain, écologiquement équilibré, libre de pollution et en harmonie avec la nature ${ }^{27} \gg$. [Notre traduction.] En second lieu, ils apparaissent sous la forme d'obligations à la charge de l'État. Ainsi, peut-on évoquer l'article 83.6 :

Constituent des devoirs et responsabilités des Équatoriennes et Équatoriens, sans préjudice d'autres prévus par la Constitution ou la loi : [...] Respecter les droits de la nature, préserver un environnement sain et utiliser les ressources naturelles de manière rationnelle, durable et responsable ${ }^{28}$. [Notre traduction.]

En troisième lieu, ces agencements normatifs peuvent se lire comme des dispositifs se référant à la nature comme objet d'application d'un principe, à l'image de l'article 275, logé dans le titre 6 portant sur le régime de développement :

le buen vivir requerra que les personnes, communautés, peuples et nationalités jouissent effectivement de leurs droits et exercent des responsabilités dans le cadre de l'interculturalité, du respect de leur diversité et de la vie en harmonie avec la nature ${ }^{29}$. [Notre traduction.]

Ou à l'instar de l'article 277.1 : «pour la réalisation du buen vivir, seront considérés comme des devoirs généraux de l'État : garantir les droits de personne, des collectivités et de la nature ${ }^{30} »$. [Notre taduction.] En dernier lieu, certaines dispositions constitutionnelles se présentent à partir de l'énonciation d'un objectif mis à la charge de l'État, comme le fait l'article 276.4 :

le régime de développement aura les objectifs suivants : récupérer et conserver la nature et maintenir un environnement sain et durable qui garantisse aux personnes et collectivités un accès équitable, permanent et de qualité à l'eau, l'air, le sol, et aux bénéfices des ressources du sous-sol et du patrimoine naturel ${ }^{31}$. [Notre traduction.]

Constitución del Ecuador, supra note 22, arts 71-74.

Ibid, art 66.7 .

28 Ibid, art 83.6 : Il nous faut tenter en langue française d'établir une différence entre les mots espagnols «sostenible » et "sustentable », à l'instar du "Vocabulaire Panlatin du Développement Durable », édité par l'Office québécois de la langue française, 2015.

29 Ibid, art 275.

$30 \quad$ Ibid, art 277.1.

$31 \quad$ Ibid, art 276.4. 
Ou à l'image de l'article 283: «le système économique est social et solidaire; il reconnaît l'être humain comme sujet et fin; tend à une relation dynamique et équilibrée entre société État et marché, en harmonie avec la nature ${ }^{32}$ ». [Notre traduction.] La typologie n'est assurément pas parfaite, ni même exhaustive, comme le montre l'article 395 qui présente une série de principes environnementaux qui semblent mélanger des mécanismes de faveur (en cas de doute sur l'atteinte des dispositions légales en matière d'environnement, celles-ci s'appliquent dans le sens le plus favorable à la protection de la nature), sur l'obligation de transversalité des obligations en matière d'environnement qui pèsent sur l'État, les autres personnes publiques et les personnes privées, ou qui semblent instaurer des normes de contrainte quant aux consultations nécessaires aux populations affectées par des impacts environnementaux à la suite de projets.

\section{B. Un nouveau constitutionnalisme ? Entre nouveaux textes et nouveaux pouvoirs du juge}

Les théories et doctrines qualifiées de néo-constitutionnalisme, de nouveau constitutionnalisme latino-américain, ou même de constitutionnalisme pluriculturel et plurinational ${ }^{33}$, partagent, malgré les diverses dénominations, des bases communes qu'il convient de présenter ${ }^{34}$. Nous adopterons aussi le terme de néoconstitutionnalisme comme mot d'usage. Le terme est revendiqué pour une bonne part de la doctrine et par celle qui s'en revendique, bien qu'il ait été formulé à la vérité par ses contradicteurs ${ }^{35}$. En effet, la dénomination de néo-constitutionnalisme est surtout le fait de la juriste italienne Susanna Pozzolo ${ }^{36}$, professeure formée à l'école du réalisme de l'université de Gênes et se revendiquant d'une approche positiviste méthodologique et de philosophie analytique. Ses travaux, notamment autour de la dénomination et de la qualification des éléments d'identification des thèses néo-constitutionnalistes, ont ouvert la voie à d'autres chercheurs et ont donné lieu à d'importantes productions académiques. L'une des premières difficultés que rencontrent les thèses du nouveau constitutionnalisme tiennent à l'importante diversité des auteurs qu'elles revendiquent pour références intellectuelles et

\section{Ibid, art 283.}

\section{Voir Sosoe, supra note 3.}

34 Nous esquisserons les principales idées fortes des théories, sans qu'il ne nous soit possible dans le cadre de cet article d'entrer dans les subtilités sémantiques et philosophiques de chaque vertente.

35 Mauro Barberis nous indique que « [c] oupable de la genèse du concept, avec Susanna Pozzolo et Paolo Comanducci, je devrais essayer de le défendre; mais je suis tenté de l'abandonner à son destin pour au moins deux raisons. Tout d'abord, nous avions inventé le mot néoconstitutionnalisme surtout afin de le critiquer; et il est assez paradoxal qu'aujourd'hui un nombre croissant de théoriciens des pays "latins" $\mathrm{du}$ Vieux et du Nouveau Continent se rangent sous cette étiquette douteuse, inconnue dans le reste du monde. Mais, surtout, les doutes de Guastini sont tout à fait fondés; si le "néoconstitutionnalisme" continuait notamment à être défini comme je l'ai défini moi-même jusqu'ici, il pourrait tout à fait s'agir d'un concept vide, d'une théorie qui n'a jamais été soutenue par aucun auteur en chair et en os voire, pire encore, il pourrait s'agir d'un concept mal formé, qui se contredit du fait même de sa définition ». Mauro Barberis, " Le néoconstitutionnalisme existe-t-il?», (2015) 25 Revue de théorie constitutionnelle et philosophie du droit 101 à la p 102.

36 Susanna Pozzolo, Neoconstituzionalismo e positivismo giurudico, Torino, Giappichelli, 2001. 
conceptuelles. Daniel Sarmento trouve les mots adéquats pour évoquer la complexité des sources, acceptions et significations du terme. Selon lui, les adeptes du néoconstitutionnalisme dans leur recherche d'un fondement à cette «théorie » s'appuient sur une diversité d'auteurs dont les positions sont assez hétérogènes, comme :

Ronald Dworkin, Robert Alexy, Peter Häberle, Gustavo Zagrebelsky, Luigi

Ferrajoli et Carlos Santiago Nino, aucun d'eux ne se définit aujourd'hui ou s'est déjà défini par le passé comme néo-constitutionnaliste. Aussi bien chez ces auteurs, comme d'autres qui se présentent comme néoconstitutionnalistes, on peut constater une grande diversité de positions jusphilosophiques et de philosophie politique : il y a des positivistes et des non positivistes, des défenseurs de la nécessité de l'utilisation de méthode dans l'application du droit et de farouches opposants de l'emploi de quelque méthode que ce soit quant à l'herméneutique juridique, des adeptes du libéralisme politique, des communautaristes, des procéduralistes. Dans ce cadre, il n'est donc pas aisé de définir le néo-constitutionnalisme, peut-être parce qu'il [...] n'existe pas un unique néo-constitutionnalisme qui corresponde à une conception théorique riche, claire et cohérente, mais plutôt diverses visions sur le phénomène juridique contemporain qui gardent entre elles quelques dénominateurs communs pertinents et qui justifient qu'elles soient regroupées sous une même étiquette, bien que rendant difficiles la possibilité d'une conceptualisation plus précise ${ }^{37}$. [Notre traduction.]

Cette extrême diversité de sources, de significations, de risques et de possibilités renforce le caractère flou du néo-constitutionnalisme ${ }^{38}$. Pour certains auteurs la nouvelle théorie constitutionnelle en question serait la conséquence d'évolutions politiques et sociales qui débouchent sur ces processus de constitutions transformatrices au Venezuela (1999), en Équateur (2008) et en Bolivie (2009). Ces pays justifient ces changements de constitutions comme conséquences des mouvements des organisations de la société civile et des communautés indigènes, parlant ainsi de refondation de l'État, sur tous les plans : politique, juridique, social, économique, culturel, idéologique et philosophique. Dans le cas de l'Équateur et la Bolivie, cette refondation est intimement et nettement liée à la valorisation des peuples ancestraux, de leur histoire et de leurs coutumes ${ }^{39}$. Dans une perspective historique, les adeptes et défenseurs des théories du néo-constitutionnalisme présentent leur thèse comme étant le résultat d'une (r)évolution du passé constitutionnel. Selon eux, il convient initialement de parler de constitutionnalisme

37 Daniel Sarmento, «O neoconstitucionalismo no Brasil: riscos e possibilidades » (2009) 3:9 Revista Brasileira de Estudos Constitucionais 1 aux pp 2-3.

38 Riccardo Guastini souligne que : «[s]elon une opinion assez répandue, ce qu'on appelle le 'néoconstitutionnalisme', serait une nouvelle philosophie du droit, avec sa propre théorie du droit, sa méthodologie, son idéologie. À mon avis, il s'agit plutôt d'un conglomérat (aux contours flous) de positions axiologiques et de thèses normatives, parmi lesquelles une thèse proprement théorique et susceptible d'être discutée a du mal à émerger. » Riccardo Guastini, "Sur le néo-constitutionnalisme » dans Jean-Yves Chérot et al, Le Droit entre autonomie et ouverture : Mélanges en l'honneur de JeanLouis Bergel, Bruxelles, Bruylant (Penser le droit), 2013, 255 à la p 255 [Guastini].

39 Qui justifie le recours aux concepts de Pachamama (terre mère ou madre tierra), de Buen-Vivir, Bien Vivir ou Sumak Kawsay. 
libéral-bourgeois, puis de constitutionnalisme social au début du $20^{\mathrm{e}}$ siècle (Mexique, République de Weimar) et finalement de nouveau paradigme néo-constitutionnel post-moderne et post-positiviste. L'idée est communément admise tant chez les néoconstitutionnalistes que pour leurs détracteurs, que les premières constitutions étaient toutes empiriques à défaut d'avoir été formalisées ${ }^{40}$. La critique qu'exercent les néo/nouveaux constitutionnalistes est surtout dirigée contre les constitutions libérales bourgeoises européennes de la fin du $18^{\mathrm{e}}$ siècle. Celles-ci auraient mis fin à l'absolutisme monarchique et instauré une société valorisant l'octroi de droits individuels promus par le droit civil que le constitutionnalisme social du début $\mathrm{du} 20^{\mathrm{e}}$ siècle aurait tenté de tempérer. Les nouveaux constitutionnalistes essaient de se démarquer des néo en ce qu'ils prétendent fonder un nouveau constitutionnalisme latino-américain stigmatisant l'incapacité des diverses catégories de constitutions antérieures (y compris les constitutions de type néo) dans le changement politique fait de pratiques autoritaires et de profondes inégalités sociales ${ }^{41}$. Le nouveau constitutionnalisme serait né à partir de la promulgation des constitutions brésilienne de 1988, colombienne de 1991, péruvienne de 1993 et vénézuélienne de 1999, mais que le moment réellement authentique serait apparu avec les constitutions de l'Équateur et de la Bolivie, respectivement en 2008 et $2009^{42}$. Ces divergences de recoupage historique et de confusion sur les qualifications théoriques à donner aux diverses constitutions par les propres auteurs des mouvements néo et nouveaux constitutionnalistes nous amènent à penser qu'il convient de synthétiser les quelques paramètres de théorie du droit qui sont revendiqués par les deux tendances éloignant ainsi toute référence géographique, ethnique, mythique ou anticoloniale ${ }^{43}$.

Synthétiquement, on peut essayer de présenter les axes du néoconstitutionnalisme ${ }^{44}$, à partir de la présentation qui en est faite par Riccardo Guastini qui énonce quelques points plus que cardinaux. Nous en présentons quelques-uns de manière descriptive : la supériorité axiologique de la constitution sur la loi; la supériorité axiologique des principes sur les règles; la supériorité axiologique des normes qui confèrent des droits sur celles qui organisent les pouvoirs publics ; l'idée qu'il y a une stricte connexion entre le droit et la morale; l'idée que le droit, à

40 Ces constitutions étaient alors coutumières, non écrites, non codifiées.

41 Roberto Viciano Pastor et Rubén Martinez Dalmau, «Fundamento teórico del nuevo constitucionalismo latinoamericano » dans Roberto Viciano Pastor, dir, Estudios sobre el Nuevo Constitucionalismo latinoamericano, Valencia, Tirant Lo Blanch, 2012, 11 aux pp 11-17.

42 Antonio Carlos Wolkmer, «Pluralismo crítico e perspectivas para um novo constitucionalismo na América Latina » dans Milena Petters Melo, dir, Constitucionalimo Latino-Americano: tendências contemporâneas, Curitiba, Juruá Editora, 2013, 19 aux pp 19-42; Raquel Yrigoyen Fajardo, « Hitos del reconocimiento del pluralismo jurídico y el Derecho indígena en las políticas indigenistas y el constitucionalismo andino " dans Mikel Berraondo, dir, Pueblos indígenas y derechos humanos, Bilbao, Universidad de Deusto, 2006, 537.

43 Il convient toutefois de ne pas exclure la forte proximité de ces tendances. Nous avons choisi l'expression néo-constitutionnalisme comme référence pour notre article. Pour le reste, les autres tendances, qu'elles se proclament du nouveau constitutionnalime en revendiquant ou non des aspects latino-américains, décolonisateurs, de pluralisme juridique, de plurinations, ou imbibées de références à des notions amérindiennes sont toutes axées minimalement autours de quelques paramètres.

44 Pour reprendre le titre de l'ouvrage de Miguel Carbonell et al, Neoconstitucionalismo(s), Madrid, Trotta, 2003. 
condition qu'il soit juste, doit être obéi ; la thèse de la connexion entre le droit et la justice; ainsi que la thèse selon laquelle les principes, à la différence des règles, s'appliquent non par voie de subsomption, mais par pondération (de sorte que l'application d'une constitution est une chose différente et irréductible à la commune application des lois).

Luis Alberto Barroso, l'une des voix qui a contribué à la divulgation du néoconstitutionnalisme au Brésil et aujourd'hui membre de la Cour suprême brésilienne, retient essentiellement trois axes dans sa définition des thèses néo, soit la reconnaissance de la force normative de la constitution, l'expansion de la juridiction constitutionnelle et une nouvelle interprétation constitutionnelle. En effet, au lendemain de la Seconde Guerre mondiale, les constitutions européennes étaient considérées comme des textes de nature essentiellement politique, dotés d'une force symbolique et d'une valeur programmatique. À cette même époque, les cours constitutionnelles commencent à se faire jour et acquièrent progressivement une autonomie juridictionnelle sur le contrôle de la loi afin notamment de protéger les droits fondamentaux. Le corollaire, selon les thèses néo-constitutionnalistes des deux premiers aspects, consiste en l'utilisation par le juge suprême des principes considérés comme des dispositions constitutionnelles supposément dotées d'une faible densité normative et de les distinguer des règles. La collision entre deux principes serait résolue par la technique dite de pondération, notamment perfectionnée par le juriste allemand Robert Alexy ${ }^{45}$.

Les critiques portées aux thèses du néo-constitutionnalisme ne manquent pas. Elles émanent de juristes de divers continents, de différents courants de pensée, mais ayant tous pour référence une approche positiviste-méthodologique du débat ${ }^{46}$. Ces critiques portent sur quelques points majeurs que nous pouvons synthétiser. L'existence d'une différence entre règles et principes est contestée, la connexion entre le droit et la morale est précisée et l'existence même d'une nouvelle conception constitutionnelle est combattue. Ainsi, Dimitri Dimoulis conteste, à partir d'un point de vue historique, le fait que les cours constitutionnelles modernes n'aient pu avoir qu'un rôle symbolique, aussi bien dans leur théorisation que dans leurs pratiques. Citant les cas des cours constitutionnelles norvégienne et grecque, qui sans prévision expresse ont su exercer un contrôle juridictionnel, à l'image de la Cour suprême américaine, qui n'est qu'un cas isolé, comme on peut trop souvent le lire ${ }^{47}$. Se référant à l'histoire des idées politiques et des conceptions constitutionnelles italienne et

45 Robert Alexy, «On Balancing and Subsumption: A Structural Comparison» (2003) 16:4 Ratio Juris 443 [Alexy, «Balancing and Subsumption »]; Robert Alexy, The Argument From Injustice : A Reply to Legal Positivism, Oxford University Press, 2002.

46 Sur les trois formes de positivisme (légaliste, comme théorie du droit ou épistémologique/méthodologique) voir Norberto Bobbio, Giusnaturalismo e positivismo giuridico, $2^{\mathrm{e}}$ éd, Milano, Edizioni di Comunità, 1972; Norberto Bobbio, Teoria generale del diritto, Torino, Giappichelli, 1993; Norberto Bobbio, Il positivismo giuridico, Torino, Giappichelli, 1996.

47 David Deener, «Judicial review in modern constitutional systems » (1952) 46:4 The American Political Science Review 1079; Dimitri Dimoulis, « A introdução do controle de constitucionalidade difuso na Grécia»(2003) 1 Revista Brasileira de Direito Constitucional 213, cités dans Dimitri Dimoulis, «Neoconstitucionalismo e moralismo jurídico » dans Daniel Sarmento, Filosofia e teoria constitutional, $3^{\mathrm{e}}$ éd, Rio de Janeiro, Lumen Juris, 2011 [Dimoulis, « introdução »]. 
française, l'auteur justifie avec une certaine pertinence la volonté qu'il y eut de doter les constitutions d'une force normative depuis au moins la fin du $19^{\text {e }} \operatorname{siècle~}^{48}$. Au moyen d'arguments tirés de l'histoire du droit, du droit comparé et usant de la méthode analytique du langage, il démontre pertinemment que le contrôle de la constitutionnalité n'était pas uniquement diffus (notamment aux États-Unis) puisqu'en combinant l'activité juridictionnelle de conformité à la Constitution avec la règle du stare decisis, la Cour suprême des États-Unis s'est implicitement dotée d'un contrôle concentré. Finalement, l'auteur démontre qu'on ne peut opposer une phase historique dénommée d'État légal qui aurait surgie au début du $20^{\mathrm{e}}$ siècle avec une phase postérieure à la Seconde Guerre mondiale qualifiée d'État constitutionnel ${ }^{49}$. Le respect de la Constitution est de l'apanage de la grande majorité des États et ce depuis l'apparition de constitutions écrites, qu'il soit explicite ou implicite, qu'il soit juridictionnellement contrôlé par voie diffuse ou exercé de façon concentrée. Un autre aspect de la critique formulée par Dimitri Dimoulis, rejoint ici par les tenants des écoles réalistes italienne et française, porte précisément sur le rôle du juge de la constitution. Comment démontrer que celui-ci serait naturellement un meilleur garant du respect du texte que le pouvoir législatif? Ne conviendrait-il pas alors de parler d'État judiciaire plutôt que d'État constitutionnel ? Ce sont aujourd'hui les axes les plus discutés de la critique du néo-constitutionnalisme ${ }^{50}$. Dans un souci de donner plus d'efficacité aux droits fondamentaux trop souvent maltraités par les pouvoirs exécutifs latino-américains, l'idée centrale du néo-constitutionnalisme consiste à s'appuyer sur les principes contenus dans la constitution (et qui souvent présentent une connexion contingente avec des valeurs morales ${ }^{51}$ ) afin de passer outre une mauvaise application d'une règle de droit.

Le concept de défaisabilité (defeasibility) est revendiqué par les néoconstitutionnalistes pour légitimer et rendre licite une non-application d'une règle de droit valide pour deux motifs. Le premier pose le postulat (avec lequel nous sommes d'accord) de l'impossibilité pour la règle de droit de prévoir toutes les situations concrètes pour lesquelles elle a fonction de s'appliquer. Certaines de ces situations nécessitent, selon l'opinion du juge, que la règle ne puisse pas être adaptée au cas concret pour des motifs de moralité (commettre une injustice ou favoriser des situations indignes). Le deuxième motif consiste à se référer à un principe constitutionnel (le plus souvent la dignité humaine, mais pas exclusivement) pour valider et justifier la non-application de la règle ; on parle alors de « défaisabilité $»^{52}$.

48 Dimoulis, « introdução », supra note 47.

49 Francisco Laporta s'interroge sur le sens donné au caractère nouveau de ces théories, et préfère concevoir la Constitution comme un genre de l'espèce Loi, confirmant également ses doutes quant à l'aspect supposément nouveau du néo-constitutionnalisme. Manuel Atienza, «Imperio de la ley y constitucionalismo: Un diálogo entre Manuel Atienza y Francisco Laporta » (2009) 31 Isonomia. Revista de Teoria Y Filosofia del Derecho 307.

50 Pierre Brunet, « La constitutionnalisation des valeurs par le droit » dans Stéphanie Hennette-Vauchez, Jean-Marc Sorel, dir, Les droits de l'homme ont-ils constitutionnalisé le monde? Paris, De Boeck, 2011, 283.

51 Ibid.

52 Sur le théme de la défaisabilité, on peut se réfèrer à la stimulante thèse de Mathieu Carpentier, Norme et exception : Essai sur la défaisabilité en droit, thèse de doctorat en philosophie, Paris 1, 2013. 
La possibilité de défaire un principe est controversée au sein des doctrines des divers ordonnancements juridiques en raison de la structure sémantique du principe. Ce dernier ne serait pas en effet susceptible d'exception (donc de défaisabilité) puisqu'il ne s'applique pas à partir d'une subsomption ${ }^{53}$. Dans ce cas, la technique utilisée est celle de la pondération : le juge choisit, en cas de collision de principes, celui qui doit s'appliquer, au moyen de méthodes tenues comme scientifiques pour certains ${ }^{54}$, mais qui ne sont à la vérité que l'expression d'un choix de valeurs morales ${ }^{55}$. Le juriste néo-constitutionnaliste le revendique et le juriste réaliste le reconnaît comme un élément de validité de sa thèse Le droit est ce qu'en font les autorités authentiques, il ne préexiste pas à la décision et toutes les théories et méthodes mobilisées dans le raisonnement argumentatif ne sont que des justifications du pouvoir de l'interprète. On perçoit alors le danger qui guette les théories néo-constitutionnalistes, celui du moralisme. C'est aussi pour cela que les réalistes (européens et parfois latinoaméricains) s'attaquent à ce qu'ils considèrent finalement comme un retour dans le passé et qualifient au passage le néo-constitutionnalisme de rétro ${ }^{56}$ ou paléoconstitutionnalisme ${ }^{57}$. Basé sur une conception empirique qui considère le droit avant toute chose comme un fait, le réalisme européen contemporain adopte pour méthodes les outils de la philosophie analytique. Il se refuse à toute possibilité d'admission de discours objectifs sur les valeurs, prônant ainsi un anti-cognitivisme éthique, qui permettrait par un métadiscours sur le droit, l'accomplissement d'une science du droit. De nature évidemment descriptive, ce point de vue reproche au nouveau constitutionnalisme sa posture normative incompatible avec la possibilité de se considérer comme une métathéorie du droit. Parce qu'il ne parvient à s'élever à la condition de ce discours externe, le néo-constitutionnalisme persiste à n'être finalement qu'une «théorie» doctrinale du droit, teinté de jus-naturalisme et présentant le danger de l'attribution d'un pouvoir moralisateur au juge constitutionnel. Ainsi, comme l'affirme pertinemment un auteur :

Le néo-constitutionnalisme n'est pas [qu']une nouvelle version du constitutionnalisme, c'est simplement une doctrine différente qui ne croit pas en la Constitution comme un texte politico-juridique susceptible de garantir le libéralisme et la démocratie, et qui fait seulement confiance aux juges des cours constitutionnelles pour protéger les individus contre les gouvernants que ceux-ci ont désignés, voire contre les individus euxmêmes. [...] pour cela, nous n'avons sans doute pas besoin de théories qui nous rassurent comme le néo-constitutionnalisme : elles nous rassurent, car elles occultent comment le droit fonctionne; mais parce qu'elles occultent comment le droit fonctionne, elles ne sont d'aucun intérêt pratique et ne peuvent que nous induire en erreur sur la nature réelle des problèmes ${ }^{58}$.

\footnotetext{
53 Riccardo Guastini démontre pourtant pertinemment que l'application d'un principe juridique peut se faire à partir de la subsomption. Guastini, supra note 38 .

54 Alexy, «Balancing and Subsumption », supra note 45.

55 Eric Millard, «Quelques bonnes raisons de douter du néo constitutionnalisme » dans Véronique Champeil-Desplats et Jean-Marie Denquin, dir, La démocratie, du crépuscule à l'aube, Paris, Mare et Martin, 2017 [Millard].

56 Ibid.

57 Dimoulis, « introdução », supra note 47.

58 Millard, supra note 55.
} 
Le réaliste, dans sa quête de vérité, ou pour le moins de description du réel, dispose alors de deux options. Se tourner logiquement vers la sociologie ou œuvrer à sophistiquer le réalisme ${ }^{59}$. Il nous paraît nécessaire, dans le cadre de notre étude, de devoir vérifier si le nouveau constitutionnalisme équatorien peut rendre compte de pratiques nouvelles et conformes au projet souhaité. Sans qu'il soit question d'analyser ces pratiques de manière exclusivement sociologique, il nous faut procéder à un premier examen des décisions judiciaires des institutions équatoriennes saisies de cas de protection de la nature.

\section{Protection de la nature à l'épreuve du contentieux judiciaire et des contingences politiques : l'enseignement de la pratique}

Le contentieux apprécié par les autorités judiciaires équatoriennes depuis l'entrée en vigueur de la Constitution de 2008 est relativement peu nombreux. Comment interpréter cette faible production de décisions de justice ? Sans nul doute la question de l'évaluation du droit est une activité délicate. Relevant, à notre avis, de la statistique ainsi que de la sociologie judiciaire, il faut toujours se garder d'aborder ces questions sans méthodologie rigoureuse. Nous savons ainsi depuis les travaux de Friedman et Percival du mouvement des litigations studies, qu'il convient de distinguer par exemple les variables empiriques liées aux procédures ou aux droits substantiels. De même pour la question qui nous intéresse ici, du taux de recours à l'institution judiciaire, il faut davantage s'intéresser à la nature des procès et des demandes plutôt que de se risquer à pratiquer une comparaison globale $^{60}$. Conséquemment, nous ne tenterons pas ici de procéder à une évaluation du droit en vigueur issu de l'activité juridictionnelle. Nous savons depuis les travaux du Centre de Recherche Critique sur le Droit de l'Université de Saint-Étienne ${ }^{61}$ que l'évaluation du droit est avant tout une activité scientifique qui nécessite sans nul doute qu'on s'interroge

sur le type de données et les niveaux d'observation pertinents, ainsi que sur les catégories d'usagers du droit associées à des catégories de normes mobilisées, sachant que les dispositifs juridiques définissent souvent en

59 Pierre Brunet remarque au sujet des thèses réalistes que : «[leurs] prolongement, ou le point d'aboutissement de certaines d'entre elles, fut la consécration d'une approche sociologique du droit qui s'est ensuite réclamée de diverses filiations. Il peut aujourd'hui paraître quelque peu contradictoire de se réclamer du réalisme sans adopter la sociologie du droit qui semble aller de pair. Pourtant, nombre de théoriciens du droit aujourd'hui se disent réalistes sans abandonner le point de vue du juriste au profit de celui du sociologue ». Pierre Brunet, « Quand le droit compte comme texte » (2013) 70:1 Rev interdiscipl ét jur 54 à la p 57.

60 Lawrence Friedman et Robert Percival, « Tales of a two Courts Litigation in Alameda and San Benito Counties » (1976) 10 Law and Soc’y Rev 267, cité par Romain Melot et Jérôme Pélisse, « Prendre la mesure du droit : enjeux de l'observation statistique pour la sociologie juridique » (2008) 2:69 Dr et Soc 331 [Melot et Pélisse].

61 Antoine Jeammaud et Évelyne Serverin, « Évaluer le droit » (1992) DS / D 263 à la p 266 [Jeammaud et Severin]. 
amont les catégories d'acteurs susceptibles d'être observés a posteriori dans des situations (contentieuses ou non) de mobilisation de la règle ${ }^{62}$.

Cette démarche de sociologie des activités judiciaires d'inspiration wébérienne ne présente pas, dans le contexte qui est le nôtre, suffisamment de matière susceptible d'être traitée. Sans doute sera-t-il possible de mener à bien une telle étude à moyen terme. Notre démarche prétend conforter notre hypothèse de départ qui consiste simplement à réfuter qu'il pourrait y avoir une différence de fonction ou de méthode entre les activités juridictionnelles (qu'elles soient issues du droit équatorien ou de bien d'autres pays) et que les diverses théories qui sont mobilisées quant au choix d'une telle interprétation le sont pour des motifs de justification.

Nous traiterons des deux premiers cas de contentieux qui furent analysés par la Cour suprême équatorienne (A) avant de vérifier notre hypothèse jusqu'au traitement décisionnel donné, non pas par une autorité juridictionnelle, mais par le propre pouvoir exécutif en conformité (formelle) avec le texte constitutionnel (B).

\section{A. Une protection judiciaire soumise à des règles processuelles « ouvertes $»^{63}$}

La Constitution de 2008 innove, sans nul doute, quant à la flexibilité processuelle qu'elle semble proposer. Trois types d'actions judiciaires sont offerts aux «justiciables ».

L'action de protection (acción de protección), l'action d'inexécution (acción de incumplimiento) et les mesures conservatoires (medidas cautelares) sont prévues par la Loi organique de garanties juridictionnelles et de contrôle constitutionnel ${ }^{64}$ (Loi organique). L'action de protection (chapitre 3 de la loi) permet une application corrective directe de droits constitutionnels. Elle est utilisée lorsqu'il y a violation des droits constitutionnels commis par des actes ou par des omissions émanant d'autorités administratives ou judiciaires, ou contre des politiques publiques qui affecteraient la jouissance ou l'exercice des droits constitutionnels. La Loi organique prévoit également au sein de l'article 41 les cas de violations de droits constitutionnels commises par des personnes privées ayant reçu délégation de service public et autres cas similaires, notamment lorsque la prestation a pu causer un dommage.

L'action d'inexécution prévue au chapitre 7 de la Loi organique permet de garantir l'application des normes du système juridique, ainsi que l'exécution des sentences, décisions et informations des organismes internationaux de protection des

62 Melot et Pélisse, supra note 60 aux pp 339-40: Les auteurs se réfèrent directement aux travaux des auteurs stéphanois, Jeammaud et Serverin, supra note 61.

63 Nous entendons par « ouvertes », un renvoi imagé à l'expression de Hart « texture ouverte ». Herbert Hart, $O$ conceito de direito, $3^{\mathrm{e}}$ éd, Lisbone, Fundação Calouste Gulbenkian, 2001 à la p 148. Nous désignons aussi l'idée par laquelle les règles processuelles sur l'intérêt à agir nous paraissent plus ouvertes dans le sens propre du mot, c'est-à-dire plus souples, de plus ample accès pour les citoyens.

64 Uruguay, Ley orgánica de garantías jurisdiccionales y control constitucional de 22 de octubre del 2009, Registre officiel $n^{\circ} 52,2^{\mathrm{e}}$ supp aux pp 32-36. 
droits humains. Encore faut-il que ces dernières contiennent des obligations claires, expresses et exigibles de faire ou de ne pas faire.

Les mesures conservatoires ont, selon 1'article 26 du chapitre 2, pour objet d'éviter ou de faire cesser les violations ou menaces de violation des droits reconnus par la Constitution et par les instruments internationaux de protection des droits humains. Elles peuvent être ordonnées conjointement ou indépendamment des actions constitutionnelles.

L'action de protection ainsi que les mesures conservatoires sont de la compétence des juges de droit commun de première instance et des cours provinciales en deuxième instance. L'intérêt à agir est très ample : la Constitution prévoit, comme cité antérieurement, au sein de l'article 71 - répété par l'article 86.1 - que toute personne, communauté, peuple ou nationalité, pourra exiger de l'autorité publique l'accomplissement des droits de la nature. Il convient de noter qu'il n'existe pas encore de législation infraconstitutionnelle protectrice de la nature. Constitutionnalisme latino-américain oblige, le droit substantiel susceptible d'être mobilisé et appliqué est issu de dispositions constitutionnelles d'application directe par le truchement des juges de première et deuxième instances, sans qu'il soit obligatoire de passer par l'examen de la Cour suprême.

C'est donc ce récent contentieux qu'il nous faut examiner à partir des caractéristiques ci-dessus mentionnées.

La première affaire examinée par les tribunaux équatoriens est assez simple sur le plan de la motivation ainsi que du droit substantiel, malgré un " cafouillage » procédural de compétence judiciaire. En l'espèce, en novembre 2010, neuf militants de l'écologie et de communautés indigènes, issus de plusieurs pays différents, parmi lesquels Vandana Shiva (Inde), Maria Blanco Chancoso (Équateur), Nnimmo Bassey (Nigéria), Cecilia Cherrez (Équateur), Ana Luz Valadez (Mexique) et Alberto Acosta (Équateur), ont tout d'abord saisi la Cour suprême de l'Équateur sur la base d'une «demande » (demanda), contre la compagnie anglaise British Petroleum (BP). Les faits concernent l'exploitation d'un gisement pétrolier maritime offshore sur la plateforme Deep Water, louée par la BP au large des côtes de la Floride, dans la zone économique exclusive des États-Unis. En avril 2010, l'explosion de la plateforme, suivie d'un incendie qui provoqua la mort de 11 hommes, ainsi que le déversement d'une nappe pétrolière estimée à 780 millions de litres, contaminèrent les eaux du Golfe du Mexique. En se basant sur le principe de juridiction universelle, les demandeurs établissent devant la Cour une requête (demanda) dirigée contre trois catégories de sujets : la compagnie BP, le gouvernement des États-Unis d'Amérique, les gouvernements des autres États du monde, ainsi que l'Organisation des Nations Unies, et en troisième lieu à l'humanité. Devant une demande peu habituelle, il convient de distinguer ce qui est demandé aux tiers de ce qui est reproché á la compagnie pétrolière responsable des dommages. À cette dernière la demande sollicite de la Cour des actes de restauration de l'environnement dégradé, des actes de compensation et des garanties, notamment en matière de responsabilité sociale pour que de tels faits ne se reproduisent pas. 
Il est aussi demandé au gouvernement des États-Unis de rétablir le moratoire sur l'exploitation de pétrole du Golfe du Mexique. Par ailleurs, les requérants prient la Cour suprême d'exhorter les gouvernements des États du monde et l'ONU d'incorporer dans les discussions sur le changement climatique, la biodiversité et le développement la reconnaissance des droits de la nature, ainsi que d'inciter à l'abandon progressif des exploitations pétrolières, à l'image du gouvernement équatorien, qui à cette époque avait renoncé à exploiter un riche gisement de pétrole situé dans la parc national Yasuni. Enfin, les requérants concluent leur demande en sollicitant de la Cour qu'elle appelle l'humanité à reprendre ses liens avec la terre mère, afin que ses droits lui soient reconnus et respectés.

Que penser d'une telle demande? Il s'agissait d'un véritable test sur l'opérationnalité du système voulu par la nouvelle Constitution. Sans remettre en cause le principe de juridiction universelle, qui en soi peut être discuté, la requête nous paraît accorder une croyance au droit et espérer une forte attente de celui-ci, référant ainsi à des aspects jusnaturalistes. Mélangeant divers agents juridiques (une entreprise privée, divers États et une organisation internationale gouvernementale), la demande, peu argumentée, apparaît comme fragile eu égard aux formulations contenues, qui alternent entre symbolisme militant et actions judiciaires tangibles. Parfois, il s'agit du caractère faussement naïf de certains points qui mérite d'être relevés, notamment lorsque les demandeurs souhaitent que l'affaire soit remise entre les mains d'une magistrate de la Cour suprême, connue pour ses positions favorables à la défense de la nature. À ce stade, la réponse qu'aurait pu apporter la Cour suprême aurait eu le mérite de souligner la difficulté ontologique dans laquelle elle se trouve : comment être un organe suprême d'un système judiciaire et politique moderne qui doit interpréter des énoncés textuels constitutionnels devant mobiliser des concepts ou notions de nature mythique (pachamama, sumak kawsay ou buen vivir)? Les contraintes, notamment procédurales, qui pèsent sur la juridiction suprême ne sont pas des moindres. Fort habilement, celle-ci s'est dispensée de toute réponse à la demande, puisque le mode de saisine utilisé (demanda) n'est pas prévu par la Loi organique, qui doit pourtant s'appliquer au contentieux des droits de la nature ${ }^{65}$. Cette saisine de novembre 2010 est absente des archives judiciaires de la Cour suprême et n'a pas dû donner lieu à la production d'une décision.

On peut présumer à défaut d'investigations faites sur place que les requérants furent conseillés de représenter leur demande auprès des juridictions de première instance compétentes pour appliquer des dispositifs constitutionnels, ce qui fut formalisé par l'action 0523-2012 introduite devant un juge de première instance de la région de Pichincha, basée sur l'article 71 de la Constitution, qui donna lieu à une décision de refus. Un appel fut formé devant la Corte Provincial de Justicia de Pichincha ${ }^{66}$ qui confirma la décision de rejet de première instance au motif que la

65 Edgar Fernandez Fernandez, «Les controverses autour de l'intérêt à agir pour l'accès au juge constitutionnel : de la défense du droit à l'environnement (Costa Rica) à la défense des droits de la nature (Équateur)» (2015), hors-série 22 VertigO La revue électronique en sciences de l'environnement, en ligne : <vertigo.revues.org/16214> [Fernandez].

66 [2013] 17111-2013-00002 (Cour provinciale de justice de Pichincha, première chambre des affaires civiles, commerciales, de location et résiduelles Équateur). 
juridiction et la compétence des juges de l'Équateur s'exercent exclusivement sur le territoire équatorien et qu'il est conséquemment impossible que l'institution judiciaire puisse apprécier de tels faits, même s'ils constituent une violation des droits de la nature, d'autant plus que la compagnie pétrolière visée n'a pas de domicile en territoire équatorien. L'avis de question constitutionnelle établi par la Cour suprême équatorienne qui consiste à vérifier de manière informative et statistique l'exercice d'interprétation d'énoncés constitutionnels par les juridictions de première et deuxième instance est assez sévère. La plupart des arguments de nature constitutionnelle sont infirmés ${ }^{67}$. Empreintes de réalisme, les décisions des juridictions de fond ainsi que l'avis de question constitutionnelle de la Cour suprême révèlent nettement les contraintes que doivent considérer les acteurs judiciaires, celles-ci n'étant que le produit du choix du nouveau système juridique équatorien, qui bien qu'il fasse référence à des textes ou concepts d'ordre mythique n'en demeura pas moins enchâssé dans des dispositifs et institutions modernes. Cette non-décision aura aussi permis de tester l'espace politique et instrumental du dispositif de juridiction universelle, qui n'a pas joué en faveur des intellectuels, initiateurs de la demande, et dont certains furent les inspirateurs de la Constitution, assumant même d'importantes charges et fonctions au sein de la République équatorienne.

La première affaire «sérieuse » ayant donné lieu à un contentieux judiciaire concerne le Rio Vilcabamba, fleuve équatorien de la province de Loja. La procédure repose sur la base d'une action de protection des droits de la nature formée par deux citoyens nord-américains résidant en Équateur depuis 2007, Richard Frederick Wheeler et Eleanor Geer Huddle, dont le fleuve en question approximait la propriété. Ces derniers ont subi des inondations causées par le fleuve en conséquence d'importants gravas jetés dans son lit au cours de la construction d'une route. La décision de première instance se contenta de rejeter la demande pour des motifs procéduraux tenant à un défaut de citation d'une autorité administrative supposément partie au procès. Les demandeurs obtinrent gain de cause devant la juridiction d'appel, qui précise dans sa décision les contours de la protection judiciaire des droits de la nature confirmant que l'action en protection est l'unique moyen judiciaire de le faire. La Cour affirme la possibilité de matérialiser le principe de précaution en cas de probabilité ou de certitude de danger de dommages environnementaux. Elle entend signifier également que la protection se veut intergénérationnelle et finalement confirme l'inversion de la charge de la preuve dans les litiges environnementaux.

Nous pouvons conclure sur le cas Rio Vilcabamba que si l'attribution de la qualité de sujet de droit à la nature a permis une meilleure protection, c'est surtout par l'extension de la qualité à agir qui permet des saisines plus certaines des tribunaux, bien que la décision de première instance puisse démontrer que certains juges vont en sens contraire. Le raisonnement de la Cour, même s'il évoque la nature ou pachamama, se garde d'investir le champ normatif propre à la détermination de ce que seraient les éléments caractéristiques de la pachamama ou du sumak kawsay. Lorsque les magistrats s'aventurent timidement sur ce terrain, les arguments relèvent du type de l'interprétation de la volonté de l'Assemblée constituante, et ce à partir

67 0-2203-13 JP Cour constitutionnelle Salle de sélection, dossier constitutionnel Équateur). 
d'archives des discours du président de ladite assemblée, Albert Acosta. La Cour provinciale saisit l'opportunité au détour de quelques lignes pour affirmer que la reconnaissance de la qualité de sujet de droit à la nature est une première dans toute l'humanité. Ne serait-il pas plutôt pertinent d'observer, pour ceux qui s'alignent sur cette position, que la Constitution ne fait que ramener le droit moderne équatorien à ses coutumes? Mais comment le prouver? Aussi peut-on penser que cette proclamation de la Cour résonne davantage comme l'affirmation d'une proposition propre à la modernité des systèmes juridiques contemporains sur une question qui fait son chemin depuis près de 40 ans. Pour le reste, la motivation de la Cour repose sur une interprétation classique d'énoncés textuels. C'est le cas pour le renversement de la charge de la preuve, qui est prévue à l'article 397 de la Constitution ou même encore sur la légitimité des intéressés à agir, qui découle de la simple application/interprétation de l'article 71 du texte suprême. Il en va également de la sorte quant au fait que la construction de la route se déroulait sans autorisation administrative environnementale. Que cette sentence d'appel soit allée dans un sens plus favorable à la protection de la nature nous paraît évident. Toutefois, de là à affirmer que la simple mention de sujet de droit attribuée à la nature en soit automatiquement responsable, il y a un pas que nous ne franchirons pas. Même si certains commentaires nous rappellent avec beaucoup d'intérêt que la décision ne résulte pas d'une pondération entre principes constitutionnels divergents ${ }^{68}$, il n'est pas sûr que dans le cas contraire la décision eut été différente. D'une part, il nous paraît difficile d'affirmer qu'il n'y ait pas devant la Cour des discussions autour des supposés bénéfices que la construction d'une route pourrait apporter au développement de la région. D'autre part, l'action d'établir un choix de valeurs à défendre semble être à la vérité le mode opératoire utilisé par la Cour. Quant à l'efficacité de la décision d'appel, la sociologie judiciaire semble nous ramener d'un terrain, duquel nous n'étions par ailleurs jamais sortis, puisque les demandeurs ont dû retourner devant la Cour pour réclamer l'exécution de la sentence, nous rappelant ainsi la similarité existante au sein des complexes systèmes judiciaires modernes, lorsqu'il s'agit d'appliquer le droit produit par les tribunaux et de mesurer l'effectivité de celui-ci dans sa généralité ${ }^{69}$.

$\mathrm{Au}$ lieu de dépendre du juge, il conviendrait de démocratiser la protection de la nature afin d'établir sa conception, son rôle notamment devant la société humaine, à l'instar des mots de François $\mathrm{Ost}^{70}$. Il en ressort donc qu'une société démocratique doive être appelée à se prononcer sur ce projet, qui pourrait valider un projet non anthropocentrique et considérer que la nature ait ses droits, mais comment traduire cela en concepts juridiques? La difficulté est celle-là, et pour notre part nous craignons que le recours au juge, au moyen de l'interprétation qu'il se fait des principes, ne soit pas une solution aussi démocratique qu'un dialogue réel avec les populations concernées par les projets environnementaux, allant même jusqu'à la

68 Sofia Suarez, «Los derechos de la naturaleza y sus primeras demandas en el ámbito judicial » (2013) 3 Revista Latinoamericana de Derecho y Políticas Ambientales 41.

69 Le concept d'effectivité est une notion complexe qui peut être défini comme la capacité de la règle de droit à orienter le comportement de ses destinataires, dans le sens souhaité par le législateur. Antoine Jeammaud, « La règle de droit comme modèle » (1990) Dalloz 199.

70 Ost, supra note 3. 
participation partielle ou totale au processus de prise de décision. S'en remettre à une logique judiciaire équivaut à croire que la logique des choses soit de l'ordre des choses de la logique ${ }^{71}$.

Sans qu'il nous soit possible ici de présenter le détail de l'ensemble des contentieux ayant pu être appréciés par l'institution judiciaire équatorienne, nous pouvons observer, après une étude synthétique d'autres décisions ${ }^{72}$, que bien que les procédures reposent sur un intérêt à agir très ample, les traits communs quant à la qualification des crimes et délits contre la nature ainsi que la motivation donnée par les tribunaux reposent sur un fonctionnement très classique, lorsque comparées à des systèmes de droit de type anthropocentrique. Sur le fond, il n'est pas démontré que les juges soient axiologiquement enclins à préserver la nature ${ }^{73}$.

\section{B. Une protection juridictionnelle constitutionnelle soumise aux contingences du pouvoir politique}

La zone d'Ishpingo-Tambococha-Tiputini (ITT) du Parque Nacional Yasuní, à la frontière du Pérou en région amazonienne, est l'objet depuis de nombreuses

71 Pour reprendre une phrase souvent citée par Pierre Bourdieu, qu'il attribue à Marx, mais dont la référence n'a jamais été établie : «La théorie marxiste commet une erreur tout à fait semblable à celle que Kant dénonçait dans l'argument ontologique ou à celle que Marx lui-même reprochait à Hegel : elle opère un "saut mortel » de l'existence en théorie à l'existence en pratique, ou, selon le mot de Marx, "des choses de la logique à la logique des choses" ». Pierre Bourdieu, Raisons pratiques : Sur la théorie de l'action, Paris, Seuil, 1994 à la p 26.

72 [2012] 269 (Sentencia Juzgado Segundo de lo Civil y Mercantil de Galápagos); Acción de protección por violación de los derechos del Río Blanco Tabacundo, sans référence (Pichincha). Ces deux affaires présentent la particularité d'associer des mesures conservatoires sur des atteintes à la nature causées par des travaux publics initiés sans licence environnementale. Dans le second cas, la société aurait formé un appel devant la Cour provinciale de Pichincha. Nous n'avons pas trouvé trace de contentieux référencés. Selon la presse locale, les travaux d'extractions de minerais semblent progresser. Alberto Araujo, « Río Blanco será el primer proyecto minero estratégico que entrará en producción » (1 juillet 2016), El Comercio, en ligne: <www.elcomercio.com/actualidad/rioblanco-proyecto-mineria-oroecuador.html>.

73 L'affaire du projet d'extraction minière Mirador de plus de 400 millions de tonnes de cuivre est à ce titre révélatrice. Plusieurs organisations civiles de défense de la nature et des communautés indigènes saisirent la justice sur la base d'une action de protection des droits de la nature contre plusieurs actes juridiques (contrat de concession minière à la compagnie à capital chinois Ecuacorriente et autorisation ministérielle d'exploitation). Sollicitant l'application de mesures conservatoires pour suspendre les travaux ainsi que la réalisation d'une seconde étude d'impact, la demande se base sur divers principes constitutionnels, notamment le principe de précaution afin d'éviter l'extinction de certaines espèces animales. Le tribunal de première instance conclut au rejet de toutes les demandes et de tous les moyens arguant que toutes les autorisations administratives furent accordées dans le respect du droit, et que l'étude d'impact n'avait pas démontré de risques pour la nature. Par ailleurs la sentence entend signaler formellement que les opérateurs au projet (compagnie minière et pouvoirs publics) avaient veillé au buen vivir des habitants de la région. Accessoirement, le tribunal a également considéré que l'exploitation des ressources était dans l'intérêt de la nation compte tenu du fait que tous les Équatoriens bénéficieront des retombées économiques, qui au surplus permettent le développement de l'État. Appel de cette décision fut formé, mais rejeté par Cour provinciale de Pichincha le 20 juin 2013 aux mêmes motifs. [2013] n 0038 (Vingt-cinquième Jugement de la Cour civile de Pichincha Équateur); [2013] n 0055 (Sentencia Juzgado Décimo Sexto de lo Civil de Pichincha Équateur). 
années de spéculations en termes de ressources pétrolières ${ }^{74}$. Le Parque Nacional Yasuni, de 982000 hectares $^{75}$, situé entre les fleuves Napo et Curaray, a été classé par l'UNESCO comme réserve de la biosphère en 1989. Il est habité par plusieurs communautés indigènes, dont celle des indiens Huaorani, qui fut protégée en $1999^{76}$ et dont le territoire a été déclaré zone intangible de conservation prohibant tout type d'activité extractive ${ }^{77}$. Après que d'importants gisements pétroliers furent découverts en 2001 dans le parc national, une proposition issue de la société civile ${ }^{78}$ a été remise en 2007 au récent président élu en 2006, Rafael Correa ${ }^{79}$. Dénommée «Initiative Yasuni-ITT» (Ishpingo-Tambococha-Tiputini) la proposition prévoit la nonextraction du pétrole situé au sein du Parque Nacional Yasuní afin de préserver son exceptionnelle richesse en termes de biodiversité. En échange de l'abandon de l'exploitation des hydrocarbures, le gouvernement équatorien, assisté par le Programme des Nations Unies pour le développement (PNUD), a sollicité une participation de la «communauté internationale » équivalente à la moitié de la revente du pétrole. L'argent serait reversé à un fonds spécialement créé et géré par des représentants de l'État équatorien, de la société civile, des bailleurs de fonds et du PNUD. Le fonds constituerait une garantie définitive de la non-exploitation du pétrole et affecterait ses ressources au financement de projets environnementaux visant à réduire les émissions de $\mathrm{CO} 2$, favoriser les énergies renouvelables et la lutte contre la pauvreté, sans compter que le projet permettrait d'empêcher la destruction de la biodiversité locale et de préserver les modes de vie des communautés indigènes Tagaeri et Taromenane qui vivent isolées dans cette région de l'Amazonie. L'initiative a été saluée par de nombreux pays, mais les contributions n'ont pas été à la hauteur. Ainsi ce renversement de la logique pollueur-payeur, qui d'une certaine manière a pu fonctionner dans d'autres circonstances présentant un danger pour la planète (sarcophage de la centrale nucléaire de Tchernobyl) s'est heurté à la realpolitik des États. Un autre argument qui a pu jouer en défaveur du plan équatorien tient aux réelles garanties de non-exploitation à moyen long terme, rappelant ainsi, pour le déplorer ou le conforter que le territoire est un élément fondamental de la souveraineté étatique. En soi, l'initiative est plus que louable compte tenu de la faible diversification de l'économie du pays et de la part importante que le pétrole exerce

74 Boaventura de Sousa Santos, «Épistémologies du Sud », (2011) 1:187 Études rurales 21 aux paras 45.

75 L'aire d'environ $10000 \mathrm{~km}$ carré a été classé parc national le 20 novembre 1979 par l'Équateur, Accord ministériel $n^{\circ} 332$, Registre officiel $n^{\circ}$ 69, 20 novembre 1979.

76 Équateur, Décret exécutif $n^{\circ} 552$, Registre officiel no 121, 02 février 1999.

77 Équateur, Décret exécutif $n^{\circ} 2187$, Registre officiel $\mathrm{n}^{\circ} 1,16$ janvier 2007. Il convient toutefois de remarquer que l'exploitation de pétrole au sein du parc fut concédée en 1993 par l'Accord ministériel $n^{\circ} 153$, Registre officiel $\mathrm{n}^{\circ} 324,25$ novembre 1993. La compagnie Maxus reçut la concession du Bloc 16, générant de nombreuses oppositions de la part d'acteurs de la société civile, notamment les Yasunidos, collectifs de défenseurs de l'environnement, actuellement actifs quant à la lutte pour la protection du parc.

78 Notamment Accion Ecologica avec Esperanza Martinez et la participation d'Alberto Acosta. Les Yasunidos, comme ils se qualifient, sont mobilisés depuis de longues années pour la défense des peuples indigènes, la préservation de l'environnement, et contre nombre d'entreprises du secteur minier et des hydrocarbures.

79 Sunniva Labarthe, «Yasuní-ITT en Équateur : le projet peut-il encore atterrir au niveau local ?» (2013) 4:76 Mouvements 90 au para 6. 
sur ses exportations. Le gisement est estimé à environ 840 millions de barils et représenterait une empreinte carbone de 400 millions de tonnes dégagées dans l'atmosphère après combustion, sans compter que le gisement en question (bloc 31) est situé en plein cœur du parc national. Ce qui nous intéresse, ce n'est point tant l'aspect purement politique - envisagé ou non en termes de sciences politiques ${ }^{80}$ tributaire de rapports de forces et de logiques de pouvoir, mais plutôt de démontrer à partir du terrain juridique que la mobilisation des règles de droit constitue l'essence de la juridicité ${ }^{81}$. Si l'on s'assigne une tâche critique ou si même l'on prétend déconstruire des opérations d'argumentations juridiques afin de révéler ici ou là un fonctionnement voilé, masquant des rapports de domination ou empreints de pure idéologie, il nous faut avant tout procéder à une lecture qui permette d'appréhender la construction des énoncés «juridiques", de nature constitutionnelle, légale, réglementaire et jurisprudentielle. Notre hypothèse, rappelons-le consiste à affirmer que l'attribution de la personnalité juridique à la nature n'est pas en soi une garantie d'une meilleure protection de l'environnement. Confortée en ce sens par notre approche théorique réaliste, notre analyse doit se tourner vers la pratique des délibérations de certaines autorités authentiques, appartenant à deux sphères de pouvoir différentes, l'exécutif et la juridiction constitutionnelle. Certes d'un point de vue politico-légal, l'initiative est en conformité avec la Constitution de 2008 et en phase avec le Plan National du Buen-Vivir 2009-2013 qui assigne à ce terme une fonction ambitieuse de paradigme alternatif au développement ${ }^{82}$.

En août 2013 le président Rafael Correa signe le Décret $n^{\circ} 74^{83}$ permettant l'exploitation du pétrole dans les blocs 31 et 43 . Au-delà des luttes et revendications politiques et sociales qui n'ont pas manqué d'intervenir, nous voulons insister sur la manière dont le droit a été utilisé. Le débat juridique autour de la mobilisation des ressources pour l'action en faveur ou contre l'exploitation du pétrole réside substantiellement, mais pas exclusivement, sur une possible antinomie des articles 57 et 407 de la Constitution équatorienne. Ceux-ci méritent d'être cités afin que nous puissions synthétiser les éléments de la discussion ${ }^{84}$ :

Chapitre quatre : Droit des communautés, peuples et nationalités

Art. 57.- Sont reconnus et garantis aux communes, communautés, peuples et nationalités indigènes, en conformité avec la Constitution et les pactes,

80 Ibid.

81 Et nous pensons que, tant d'un point de vue du réalisme juridique que d'une analyse en termes de sociologie empirique du droit, cette définiftion de la juridicité est la même : les règles de droit ne se mettent pas en action toute seules, la norme n'est pas le texte et ne lui préexiste pas.

82 Toute la difficulté réside sur la signification à donner au buen vivir : alternative au développement ou développement alternatif? L'enjeu, en termes de choix politiques et conséquemment juridiques, tient à cette question. L'Équateur doit en effet faire face à ce dilemme et le cas Yasuni ITT a clairement, au plus grand désespoir des contempteurs du buen vivir, choisi la seconde option. Tant que l'on puisse toutefois considérer qu'il s'agisse véritablement que l'extraction de pétrole puisse in fine être considérée comme un développement alternatif, sauf à se ranger à la pertinence de la thèse de la malédiction du pétrole.

83 Équateur, Décret exécutif $n^{\circ} 74$, Registre officiel supplémentaire $n^{\circ} 72,3$ septembre 2013 [Décret exécutif $\left.n^{\circ} 74\right]$.

84 Constitución del Ecuador, supra note 22, arts 57 et 407. 
conventions, déclarations et autres instruments internationaux des droits humains, les droits collectifs suivants :

[...] 7. La consultation, libre et informée dans un délai raisonnable à propos des plans et programmes de prospection, exploitation et commercialisation de ressources non renouvelables situées sur leurs terres et qui peuvent les affecter sur le plan environnemental et culturel ; participation aux bénéfices des projets et recevoir des indemnisations quant aux préjudices sociaux, culturels et environnementaux. La consultation que doivent réaliser les autorités compétentes sera obligatoire et opportune. Si le consentement de la communauté consultée n'est pas obtenu, il sera procédé à l'application de la Constitution et de la loi.

Les territoires des peuples en isolement volontaire sont de possession ancestrale, irréductible et intangible et il y est prohibé tout type d'activité extractive. L'État adoptera les mesures pour garantir leurs vies, faire respecter leur autodétermination et leur volonté de rester en isolement, ainsi que veiller à l'observation de leurs droits. La violation de ces droits constituera un délit d'ethnocide, qui sera légalement qualifié. L'État garantira l'application de ces droits sans aucune discrimination, en condition d'égalité et d'équité entre femmes et hommes.

$[\ldots]$

Section trois : Patrimoine naturel et écosystèmes

Art. 407.- Est prohibée l'activité extractive de ressources non renouvelables sur les aires protégées et en zones déclarées comme intangibles, y compris l'exploitation forestière. Exceptionnellement, lesdites ressources pourront être exploitées sur pétition motivée de la Présidence de la République après déclaration d'intérêt national émise par l'Assemblée Nationale, qui si elle l'estime convenant, pourra convoquer une consultation populaire.

Sans entrer dans une discussion large et complète de l'éventail des textes et énoncés législatifs et réglementaires susceptibles d'être mobilisés ${ }^{85}$, nous nous limiterons au débat suscité par les deux articles de la Constitution cités ci-dessus. L'article 57 de la Constitution permet la prohibition des activités extractives sur les territoires des peuples en isolement volontaire ${ }^{86}$ sans prévoir explicitement ou non aucune exception. À l'évidence, les acteurs menant des combats pour l'arrêt de l'exploitation misent surtout, mais non exclusivement, sur cet article pour espérer une victoire juridictionnelle. L'article 407 de la Constitution prohibe également de manière plus large les activités extractives à la condition qu'elles portent sur des ressources non renouvelables (y compris l'exploitation forestière) situées sur certains espaces protégés légalement ou déclarés comme intangibles. Au contraire de l'article 57, l'article 307 prévoit explicitement une exception conditionnée à un acte motivé à l'initiative du chef de l'exécutif - en l'espèce le Président de la République -

85 Malka Andrea San Lucas Ceballos, Los derechos de los pueblos indígenas ante la explotación petrolera del Yasuni-ITT, thèse de doctorat en droit, Université Rovira i Virgili, Tarragone, 2015 [Ceballos].

86 «Pueblos en aislamiento voluntario ». 
et dont la responsabilité est divisée avec le législatif - l'Assemblée nationale - qui a la charge de produire une déclaration d'intérêt national, pouvant opter ou non pour une consultation populaire. À la vérité il n'est pas sûr qu'il y ait une antinomie, on peut pertinemment considérer que l'exception prévue à l'article 407 n'est pas applicable puisque le gisement Yasuni-ITT est situé en terre de peuples en isolement volontaire. Ainsi, en l'espèce l'on pourrait mobiliser, au moins, deux ressources argumentatives en faveur d'une interprétation contraire à l'exploitation pétrolière ${ }^{87}$. La première est la fameuse technique positiviste, largement enseignée au cours des premiers enseignements des facultés de droit, lex specialis derogat legi generali. La seconde, prévue à l'article 427 de la Constitution équatorienne ${ }^{88}$, est qu'en cas de doute le principe de l'interprétation favorable puisse prévaloir sur toute autre interprétation :

Art. 427. -Les normes constitutionnelles s'interprètent par leur teneur littérale qui s'ajuste le plus à la Constitution dans son intégralité. En cas de doute, elles s'interprètent dans le sens qui favorise le plus la pleine vigueur de droits et qui respecte le plus la volonté du constituant et en accord avec les principes généraux de l'interprétation constitutionnelle ${ }^{89}$.

L'aspect controversé de la nature juridique de la déclaration d'intérêt national de l'Assemblée nationale a suscité de nombreuses discussions ${ }^{90}$. Est-ce un acte législatif? En cas de réponse positive, il semblerait alors que la jurisprudence antérieure de la Cour suprême soit d'exiger la consultation des populations concernées, d'autant plus que l'Équateur a spécifiquement été condamné sur ce point par la Cour interaméricaine des Droits de l'Homme (mais sous l'effet de la Constitution antérieure, étant donné que l'affaire s'est déroulée entre 2003 et 2012) (11. $^{91}$. Mais en somme, de l'argument de faveur à celui de la spécialité de la loi, ces deux arguments, considérés comme des ressources pour l'action, nous paraissent au final bien représentatifs d'un système classique et moderne de droit. De plus, nous avons quelques interrogations pour déceler les aspects de révolution ou d'évolution théorique dans la manière d'aborder le droit et de résoudre ses problèmes d'antinomies.

87 Il en va ainsi en ce qui concerne l'interprétation d'énoncés juridiques de toute une panoplie de méthodes que certains désignent sous le vocable d'herméneutique. Cette dernière couvre un vaste champ de possibilités disciplinaires (dogmatique juridique, philosophie, théologie).

88 L'article 395.4, à la section première: Nature et Environnement, logée au chapitre second: Biodiversité et ressources naturelles indique qu'en « cas de doute sur l'étendue des dispositions légales en matière environnementale, celles-ci s'appliqueront dans le sens le plus favorable à la protection de la nature ». Constitución del Ecuador, supra note 22, art 394.4. Si cet article laisse augurer une mobilisation de la part des acteurs impliqués au conflit, il peut paraître convenant, pour d'autres, de rappeler que le cas Yasuni-ITT est d'ordre constitutionnel et relève donc d'une discussion limitée au dispositif de l'article 407.

89 Constitución del Ecuador, supra note 22, art 427.

90 Ceballos, supra note 85 aux pp 324-25.

91 Pueblo Indígena Kichwa De Sarayaku (Équateur) (2013), Fondement et réparation, Inter-Am Ct HR (Sér C) n 245. 
En pratique, le combat juridictionnel a été lancé par deux actions devant la Cour suprême ${ }^{92}$. La première demande d'inconstitutionnalité déposée devant la Cour constitutionnelle ${ }^{93}$ porte sur une supposée inconstitutionnalité des actes administratifs préparatoires aux décrets présidentiels ${ }^{94}$ et de l'acte administratif sollicitant la déclaration d'intérêt national autorisant l'exploitation pétrolière. Le litige est assez technique et porte sur la zone exacte de délimitation des forages prévus par les décrets d'août 2013. La demande conclut sur l'inconstitutionnalité des normes ayant considéré que le territoire prévu est en zone intangible, mais pas en territoire ancestral de peuples en isolement volontaire, afin de ne pas faire application de l'article 57 de la Constitution. La seconde action constitutionnelle a été déposée au nom de la confédération des peuples Kichwas de l'Équateur, Ecuarunari ${ }^{95}$. Elle s'oppose principalement à l'action de la résolution de déclaration d'intérêt national de l'Assemblée nationale, qui contreviendrait expressément aux dispositions constitutionnelles, notamment sur les droits des peuples indigènes, sur le territoire des peuples en isolement volontaire.

La procédure constitutionnelle prévoit expressément que la Cour constitutionnelle doit se prononcer dans le délai de 15 jours suivant les demandes sur l'admissibilité de celles-ci ${ }^{96}$. Dans le premier cas, la décision d'admissibilité a été admise par la Cour constitutionnelle le 10 mai 2016, soit plus de deux ans et demi après le délai imparti, et sans aucune explication quant à la publication plus que tardive de la décision d'admissibilité ${ }^{97}$. Sur le fond, plus de trois ans après le dépôt de la demande, il n'existe aucune décision de la Cour. Dans le cas de la seconde demande, portant sur une action en inexécution, déposée en mai 2014, la Cour a notifiée le demandeur le 19 août 2014 pour lui signifier, qu'afin qu'elle puisse se prononcer sur la seule admissibilité de la demande, qu'il soit en mesure de compléter certains points de sa demande, en fournissant des précisions sur le type d'action constitutionnelle qu'il prétend former, ainsi que sur l'acte ou l'objet de la demande, et ce dans un délai de cinq jours. Le demandeur n'ayant fourni les éléments qu'en septembre ou novembre 2014, la Cour rejette l'action et classe le dossier le 18 décembre $2014^{98}$.

92 Nous ne mentionnons pas les autres ressources utilisées, comme l'initiative de consulta popular basée sur l'article 104 de la Constitution, déposée par un nombre de citoyens équatoriens et qui se perd dans le comptage des signatures.

93 Corte constitucional, sala de admision, [2016] n 0005-13-IA. La demande présentée par Tarquino Orellana le 5 septembre 2013. Tarquino Orellana fut le Vice-président de l'ancien Tribunal constitutionnel entre 2006 et 2007, Vice-président de la Commission pour la Réforme de la Législation Équatorienne et fut membre de la Commission de Rédaction de la Constitution de 2008.

94 Décret exécutif $n^{\circ} 74$, supra note 83; Équateur, Décret exécutif $n^{\circ}$ 63, Registre officiel supplémentaire $\mathrm{n}^{\circ}$ 63, 21 août 2013; Équateur, Décret exécutif $n^{\circ} 84$ du 17 août 2013 modifiant l'article 5 du décret $n^{\circ} 74$, Registre officiel supplémentaire $\mathrm{n}^{\circ} 77,10$ de septembre 2013.

95 Action pour inexécution, Corte Constitucional, Sala de admision, [2014] n 0037-14-AN. Déposée le 15 mai 2014 par Carlos Pérez Guartambel représentant ladite Confédération.

96 Uruguay, Ley orgánica de garantías jurisdiccionales y control constitucional de 22 de octubre del 2009, Registre officiel $\mathrm{n}^{\mathrm{o}} 52,2^{\mathrm{e}}$ supp, art 80.

97 Communiqué au demandeur, Corte Constitucional del Ecuador, [2016] oficio 2531-CCE-SG-NOT2016.

98 Corte Constitucional del Ecuador, auto de Sala de Admisión y Razón, [2014] n 0037-14-AN. La décision semble avoir été rédigée de manière assez rapide et contient au moins deux erreurs de date. La 
Conformément à notre optique d'analyse réaliste choisie, il nous faut percevoir que toutes les ressources mobilisées pour l'une ou l'autre des thèses défendues reflètent le choix de théories justificatrices d'une certaine représentation de ce qu'est, doit ou devrait être le droit. Dans les deux cas d'espèce, l'absence de respect des délais procéduraux, l'absence de motivation des décisions juridictionnelles sur le contrôle des actes de l'exécutif et la rédaction sommaire et cavalière des décisions démontrent une attitude désinvolte de la Cour constitutionnelle. Elle confine presque au déni de justice ou illustre, au mieux, un réalisme juridique crasse. Sur le plan politique, la présentation par communiqué de presse des motivations du Décret exécutif $n^{\circ} 74$ confirme un réalisme politique assumé. L'exploitation du pétrole y est conçue comme une manne financière permettant de freiner l'extension de la frontière de l' "agrobusiness » et de préserver notamment les conditions des paysans ruraux, en luttant contre la pauvreté. Le buen vivir, interprété et appliqué par les trois pouvoirs équatoriens, le conçoit somme toute comme un développement économique et social plutôt classique sans que l'on puisse déceler où serait la part d'alternative au développement.

Au terme de notre article, nous pouvons souligner que la personnification juridique instaurée par la Constitution équatorienne de 2008 ne semble pas offrir, $a$ priori, un degré de protection supérieur aux systèmes anthropocentriques ${ }^{99}$. Trois aspects permettent de conforter notre thèse.

En premier lieu, depuis un point de vue de théorie du droit, notre choix épistémologique et méthodologique d'un réalisme européen latin, et même latinoaméricain, ne nous autorise pas à déceler un possible fonctionnement du droit par le truchement simple et exclusif des énoncés. Changer de textes constitutionnels, légaux, réglementaires ne provoque pas naturellement un changement quant aux significations qui seront données à ce texte. Proclamer le néo-constitutionnalisme comme une nouvelle (méta)théorie du droit nous semble exagéré et n'est point démontré. Que la norme soit en effet une règle ou un principe, elle ne s'applique pas ou presque pas sans le truchement du tiers intervenant, l'autorité authentique chargée d'interpréter l'énoncé (juges, fonctionnaires publics, législateurs). Se voulant une théorie normative/prescriptive, le néo-constitutionnalisme ne constitue pas une théorie de la connaissance, tout au moins participe-t-il d'une doctrine. En ce qui a trait à la production de nouveaux textes supposés comme axiologiquement plus protecteurs des droits de la nature, ceux-ci peuvent pour le moins constituer des contraintes pour les

Cour prétend ainsi que le demandeur aurait introduit sa demande initiale le 15 mai 2010, au lieu du 15 mai 2014. Enfin, elle rejette sa demande comme hors-délai au motif que les compléments d'information ont été déposé « le $1^{\mathrm{er}}$ septembre de novembre 2014 ». [Notre traduction.].

99 Eu égard aux premières décisions de la Cour suprême équatorienne ainsi que des tribunaux et cours interprétant la nouvelle Constitution, et sous réserve d'interprétations futures plus « progressistes ». 
autorités authentiques interprétatives. Cela dit, l'analyse des contentieux judiciaire ${ }^{100} \mathrm{~S}$ et constitutionnels ne permet pas de révéler où il y aurait eu contrainte, compte tenu de la faible motivation des jugements ou du caractère désinvolte ou inique de certaines juridictions. Au surplus, l'office du juge nous a permis de vérifier que sa manière de produire la norme à partir du cas concret n'a pas été modifiée par les théories néo-constitutionnalistes.

En second lieu, si l'on s'éloigne des normes substantielles et que l'on s'en tient aux aspects de droit processuel, on a pu observer que la Constitution offrait sans nul doute des avancées quant à la saisine des tribunaux. L'intérêt et la capacité à agir sont des mécanismes à l'évidence incontournables quant aux avancées du droit de l'environnement, puisqu'il est nécessaire de passer par cet instrument propre aux systèmes judiciaires modernes. Néanmoins, l'analyse du contentieux nous conduit à penser que cela n'influe guère sur la substantialité des normes appliquées à l'issue des procès ${ }^{101}$. Finalement, on peut considérer que le caractère ouvert des règles processuelles permet seulement d'accroître le nombre de contentieux. Par ailleurs, si l'on examine, dans une démarche de droit comparé, certaines évolutions du droit de l'environnement d'États appartenant à des systèmes anthropocentriques, on peut noter que le degré de protection ne diffère pas et en conclure que finalement la question de l'extension de la lutte pour l'intérêt à agir ne paraît pas déterminante ${ }^{102}$. En somme, si l'on convient que la principale innovation de la Constitution tient à la reconnaissance de sujet de droit à la nature, force est de reconnaître que celle-ci a besoin du truchement de personnes physiques pour agir théoriquement en son nom ${ }^{103}$. Cet intérêt à agir élargi à tous n'exprime dès lors pas la subjectivité de la nature, mais caractérise la signification d'une volonté personnelle, conçue comme un fait qui se transforme en acte pouvant revêtir différentes dénominations (action de protection, mesures conservatoires, etc.). L'intérêt à agir élargi peut statistiquement générer une augmentation du contentieux, sans qu'il n'y ait de corrélation naturelle avec une jurisprudence « qualitativement meilleure » ou disons-le effective.

100 La plupart des articles défendant la nouvelle conception constitutionnelle de l'Équateur et le mécanisme de la personnalité juridique attribuée à la nature ne traitent généralement pas d'étude du contentieux, se contentant de promesses ou d'horizons à conquérir confirmant ainsi que le nouveau constitutionalisme n'appartiendrait pas au monde des faits, mais plutôt de l'ordre du devoir être, doté ainsi d'un fort aspect prescriptif. Pour une illustration de la rhétorique néo-constitutionnaliste, sans souci d'étude des pratiques contentieuses, voir Freitas, supra note 11.

101 Voir Mary Elizabeth Whittemore, «The Problem of Enforcing Nature's Rights under Ecuador's Constitution: Why the 2008 Environmental Amendments Have No Bite» (2011) 20:3 Pac Rim L \& Pol'y J 659.

102 Voir Fernandez, supra note 65.

103 Comme le remarque avec beaucoup d'acuité Edgar Fernandez Fernandez, « il nous semble pertinent de soulever une situation curieuse que nous avons remarquée dans tous les cas de défense des droits de la nature auxquels nous avons fait référence antérieurement. Bien que les demandeurs aient agi devant le juge, non pas à titre personnel, mais en représentation de la nature - car celle-ci est le titulaire des droits dont la défense a été invoquée -, les affaires ont été officiellement enregistrées par les cours équatoriennes comme si le demandeur ou la personne dont les droits avaient été lésés (actores/ofendidos) avait été la personne qui agit au nom de la nature, et non pas celle-ci. Par exemple, dans l'affaire du fleuve Vilcabamba, ont été enregistrés comme actores/ofendidos les époux Richard Fredrick Wheeler et Eleanor Geer Huddlee. » Fernandez, supra note 65 au para 45; Voir également Cons const, 8 avril 2011, M Michel $Z$ et autres, (2011) JO, 9 avril 2011, 6361, 2011-116 QPC qui élargit et démocratise le mécanisme de l'intérêt à agir. 
En troisième lieu, on perçoit que l'indigénisme juridique latino-américain procède à un syncrétisme, mêlant énoncés constitutionnels de types positifs et dogmatiques avec une Constitution lourde de plusieurs centaines d'articles et de mécanismes processuels classiques, bien qu'ils permettent un accès ouvert et ample aux juridictions du premier degré. Formellement, ces règles processuelles sont les mêmes pour les juridictions du second degré ou pour la juridiction constitutionnelle, il en va toutefois différemment lorsqu'on observe la pratique de l'admission des recours en appel ou devant la Cour constitutionnelle. La pachamama et le sumak kawsay ne sont, au regard des contentieux étudiés, jamais définis ou discutés intrinsèquement, mais justifiés par la mobilisation de règles classiques, semblables à celles des systèmes anthropocentriques. Cet indigénisme juridique, louable en soi, est possiblement effectif si l'on songe à des mécanismes du type de celui du pluralisme juridique avec son concept de relevance comme Santi Romano l'a proposé104. Il nous interroge cependant sur cette articulation entre coutumes et droit moderne. Sans nul doute cette question qui relève de l'anthropologie juridique mérite sans cesse d'être pensée de manière à favoriser le renouvellement des idées, notamment sur le plan théorique $^{105}$. On court toujours le risque d'une essentialisation de l'amérindien, pensé comme sujet naturellement protecteur de la nature et de se perdre dans une conception mythifiée de l'environnement, notamment d'un point de vue historique et anthropologique $^{106}$. De la même manière, l'hypothèse scientifique Gaia de James Lovelock est souvent mobilisée comme une ressource argumentative par certains néoconstitutionnalistes ${ }^{107}$ afin de tenter de conforter la thèse d'une nature qualifiée de Terre Mère (pachamama). L'hypothèse Gaia est toutefois loin de recueillir l'unanimité de la communauté scientifique et est justiciable de nombreuses critiques notamment sur son caractère téléologique, son holisme, ou son absence de réfutabilité $^{108}$. L'aspect éminemment syncrétique de la Constitution équatorienne, soucieux dans un projet de décolonisation des idées, ajouté à la recherche d'une alternative au développement (capitaliste ?) nous semble faire l'économie des mots et par conséquent de restreindre excessivement ce débat à des hypothèses fragiles ${ }^{109}$. Notre analyse critique des théories et pratiques qui sous-tendent l'approche néoconstitutionnaliste nous semble devoir être approfondie par un questionnement en termes de modèles de développement économique et social dans un contexte d'économie globalisée. Il semblerait ainsi que la projection anti-économiciste du buen

104 Santi Romano, L'ordre juridique, $2^{\mathrm{e}}$ éd, Paris, Dalloz, 2002. Le livre a été publié en italien en 1918 et édité en français seulement en 1975, date de la première édition.

105 Louis Assier Andrieu, « Penser le temps culturel du droit : Le destin anthropologique du concept de coutume » (2001) 160 L'Homme 67 à la p 89.

106 William Balée, «Qui a planté les décors de l'Amazonie ? (2000) 333 La Recherche 80.

107 Voir Freitas, supra note 11.

108 Latour, supra note 4.

109 La problématique des États du Sud doit-elle être pertinemment envisagée à partir du prisme des études postcoloniales? Les approches épistémologiques de Boaventura dos Santos ou philosophiques d'Enrique Dussel tiennent lieu de sources de références intellectuelles et théoriques pour bon nombre de juristes néo-constitutionnalistes latino-américains. Contra Jean-François Bayart, Les études postcoloniales, un carnaval académique, coll « Disputatio », Paris, Karthala, 2010. Pour une approche plus nuancée, voir les travaux de Santiago Castro Gómez, Crítica de la razón latinoamericana, $2^{\mathrm{e}}$ éd, Bogotá, Editorial Pontificia Universidad Javeriana, 2011. 
vivir soit approximative, trop utopique et peu en proie aux réalités contemporaines ${ }^{110}$. Finalement, la vision néo-constitutionnelle équatorienne de la nature sujet de droit n'est pas exempte de critiques interdisciplinaires ${ }^{111}$. Du point de vue de la théorie du droit, de la sociologie des activités judiciaires, du regard anthropologique et des fragiles conceptions économiques, l'on perçoit une certaine naïveté ${ }^{112}$ à penser le droit constitutionnel moderne à partir de coutumes, pensées et références religieuses, d'ordre mythique, tant indéterminées que les règles positives «modernes» et qui seront inéluctablement interprétées par un juge ${ }^{113}$. Conçue comme un modèle syncrétique et vertical qui part des théories pour influer sur les pratiques, cette doctrine dont l'efficacité n'est pas démontrée n'est absolument pas nouvelle et correspond à un retour à une vieille antienne ${ }^{114}$.

110 On peut philosophiquement être contre une pensée économiciste, la combattre dans ses pratiques et vouloir élaborer légitimement un projet alternatif, mais il nous semble que les expertises nécessaires au passage à la pratique n'aient pas été approfondis.

111 Voir les travaux de philosophie et de sciences sociales ainsi que les propositions de François Ost envisageant la nature comme un projet. Il convient toutefois de rappeler que la «province des îles Loyauté a adopté en avril 2016 la première phase de son Code de l'environnement, le Cepil, après trois ans de travaux et une large consultation des autorités coutumières et de la population loyaltienne. D'autres phases sont prévues pour aboutir à une version complète probablement en 2017. Une réflexion voit actuellement le jour afin de sélectionner des éléments de la nature qui pourraient se voir attribuer un statut de personne légale ». C Schoenholtzer, " La province des îles a son nouveau code de l'environnement»(25 août 2016), Demain en Nouvelle Calédonie, en ligne : <www.dnc.nc/laprovince-des-iles-a-son-code-de-lenvironnement/>.

112 Voir à ce sujet les nuances apportées par un auteur de la mouvance du nouveau constitutionnalisme, Roberto Gargarella, qui décèle en 2008 avec beaucoup de pragmatisme le risque d'un certain déficit démocratique des nouvelles constitutions latino-américaines et finit par admettre en 2014 l'aspect ancien du nouveau constitutionnalisme ainsi que ses pratiques autoritaires: Roberto Cargarella, «Cambiar la letra, cambiar el mundo » (13 octobre 2008), El País, en ligne : <elpais.com/diario/2008/ 10/13/opinion/1223848811_850215.html>; ainsi que Roberto Gargarella, «El nuevo constitucionalismo latinoamericano » (20 août 2014), El País, en ligne : <elpais.com/elpais/2014/07/ 31/opinion/1406816088_091940.html>.

113 Sans nul doute le changement de constitution ne semble pas suffisant pour changer les structures en place et les modes de pensées. L'influence de la prise en compte des textes juridiques sur le comportement des divers acteurs sociaux méritera à moyen terme de plus amples recherches d'ordre sociologique afin de vérifier la teneur de nos propos et de continuer ainsi à alimenter le débat.

114 José Sánchez Parga, « Discursos retrovolucionarios : Sumak Kausay, derechos de la naturaleza y otros pachamamismos » (2011) 84 Ecuador Debate Revista 31. 